\title{
Analysis of differential expression of genes induced by ethephon in elongating internodes of maize plants
}

\author{
Xiaoyi WEI ${ }^{1,2 *}$, Weiqiang ZHANG ${ }^{1 *}$, Qian ZHANG ${ }^{1}$, Pei SUN ${ }^{2}$, Zhaohu LI ${ }^{1}$, Mingcai ZHANG ${ }^{1}$, Jianmin LI $^{1}$, \\ Liusheng DUAN $(\bowtie))^{1}$
}

1 State Key Laboratory of Plant Physiology and Biochemistry, Engineering Research Center of Plant Growth Regulator, Ministry of Education/College of Agronomy, China Agricultural University, Beijing 100193, China

2 Xinxiang Academy of Agricultural Science, Xinxiang 453000, China

\begin{abstract}
Plant growth regulators (PGRs) are commonly used in cereal cropping systems to restrict plant height and control lodging. Ethephon has been reported to shorten internodes and increase grain yield of maize. To analyze the transcriptomic profiles of maize internode elongation following ethephon treatment, differentially expressed genes were compared between the treatment and control samples of inbred line Zong 31 using the Affymetrix Maize Genome Array. According to the microarray data, 326 probe sets showed significant change in expression. Further research revealed that the most remarkable effects of ethephon on maize internodes elongation occurred during a $48 \mathrm{~h}$ period, when 89 differentially expressed genes were detected. There were dramatic change in transcript levels at $24 \mathrm{~h}$ and six Auxin transport genes and four gibberellin biosynthesis pathway genes were differentially expressed in Zong 31 in response to ethephon treatment. In summary, we showed that gaseous ethylene release is involved in internode meristem cell elongation through the regulation of plant hormone signaling in maize. This work provides a platform for studies in which candidate genes will be functionally tested for involvement in internode elongation.
\end{abstract}

Keywords maize, ethephon, internode elongation, microarray, qPCR

Received April 11, 2016; accepted May 13, 2016

Correspondence: duanlsh@cau.edu.cn

${ }^{*}$ These authors contributed equally to the work

\section{Introduction}

Lodging (root and stalk lodging) is one of the major problems in maize production worldwide, impacting greatly on field management, grain quality, and mechanized harvesting and accounts for annual yield losses of $5 \%-25 \%^{[1,2]}$. Chemical control is an effective measure to reduce plant height and prevent lodging in maize ${ }^{[3]}$. Plant growth regulators are involved in various aspects of chemical control to promote, retard or inhibit growth, and are commonly applied in agriculture to control lodging in cereal crops ${ }^{[4]}$. These exogenously applied chemical compounds reduce elongation and lead to dwarfing by promoting biosynthesis of ethylen or inhibiting production of gibberellins (GA). Ethephon application results in the release the gaseous plant hormone ethylene, which can trigger myriad physiological and morphological responses in cereal crops. Previous studies have shown that ethylene released after applying ethephon can reduce plant height and control lodging by decreasing the internode length ${ }^{[3,5-7]}$.

Ethylene has multiple and diverse roles in plant physiology, including the triple response, inhibition of growth, stress response, fruit ripening, leaf/petal/fruit senescence, leaf abscission, promotion of stem elongation in deep water rice, sex determination, breakdown of seed dormancy/promotion of seed germination and adventitious root formation ${ }^{[8-11]}$. During the past decade, molecular genetic studies on a model dicotyledonous plant (Arabidopsis) and a monocotyledonous plant (rice) have revealed a linear signal transduction pathway from signal perception to transduction and identified the signal components involved in the ethylene biosynthesis pathway ${ }^{[12-15]}$. For example, the physical interaction between the receptors (ETR1, ERS1, ETR2, ERS2 and EIN4) and CTR1 in the absence of ethylene keeps downstream signaling compo- 
nents EIN2 and EIN3 inactive, and ethylene insensitive EIN2 and EIN3 are known to be tightly controlled by a proteasome-dependent regulatory module ${ }^{[16,17]}$. The regulation of this signaling pathway involves complex regulatory mechanisms involving regulation by protein turnover, potential posphorylation of EIN3 through MAP kinase cascades, transcriptional activation of the receptors and EIN3 binding F-box proteins and EIN5/exoribonuclease (XRN4)-mediated control of $E B F$ mRNA levels ${ }^{[18]}$. MKK9-MPK3/6, the mitogen-activated protein kinase cascades, are probably also involved in ethylene signaling and/or ethylene biosynthesis ${ }^{[19,20]}$. Additionally, as a plant hormone, ethylene is known to differentially control internode elongation by interacting with other plant hormones such as auxin (indole-3-acetic acid, IAA), GA and abscisic acid (ABA), and some molecular elements have been identified in Arabidopsis or rice ${ }^{[3-7,21-26]}$. Nonetheless, to the best of our knowledge, the signal transduction cascade and molecular mechanisms associated with ethylene biosynthesis in maize internode elongation have not been characterized in detail.

Microarray technology has been established as a sensitive and convenient tool for rapid analysis of plant gene expression to characterize different processes of plant growth and development ${ }^{[27-29]}$. Research on signal transduction pathways activated during plant responses is often initiated from microarray data analyses ${ }^{[30]}$. Recently, increasing information on microarray analysis of plant hormones that are differentially regulated during their own biosynthesis and through signal transduction pathways has been obtained ${ }^{[31-35]}$. However, no report on large-scale differential gene expression analysis of ethephon treatment affecting internode elongation in maize has been published. Considering the importance of morphological improvements in plant architecture for maize production, understanding the molecular mechanisms controlling internode elongation and development is essential ${ }^{[36,37]}$.

In the present study, we found phenotypic responses of maize internodes to ethephon, through released ethylene, included inhibition of longitudinal cell growth and promotion of lateral growth. Microarray data revealed that the greatest stabilization effect of ethephon on maize internode elongation occurs during the first $48 \mathrm{~h}$, with the peak in differentially-expressed genes in maize internodes occurring at $24 \mathrm{~h}$. Furthermore, six auxin transport genes and four GA biosynthetic pathway genes are differentially expressed in maize (Zea mays L.) inbred line Zong 31 (one of the most widely used for maize breeding in China, developed and contributed by National Maize Improvement Center of China, China Agricultural University) by ethephon treatment. Therefore, we postulated that ethylene gas release is involved in internode meristem cell elongation by regulation of the plant hormone signaling crosstalk in maize. This might provide a basis for further study of the molecular mechanisms of the phenotypic responses of maize internodes to ethephon.

\section{Materials and methods}

\subsection{Plant materials and ethephon treatment}

Seeds of maize (Zea mays) inbred line Zong 31 were sown in a greenhouse $\left(28^{\circ} \mathrm{C}\right.$ day $/ 20^{\circ} \mathrm{C}$ night; $16 \mathrm{~h}$ photoperiod; $40 \%$ RH (relative humidity)) at China Agricultural University, Beijing, in December 2009. At the nine-leaf growth stage where leaf collars were visible, ethephon $\left(270 \mathrm{~g} \cdot \mathrm{hm}^{-2}\right.$ of $\left.600 \mathrm{mg} \cdot \mathrm{L}^{-1}\right)$ was evenly sprayed on maize foliage with 200 seedlings as the treatment group and water sprayed on another 200 seedlings as the control group. All other agronomic practices were applied uniformly to both groups.

\subsection{Phenotypic and physiological characterizations analysis}

To test internode phenotypic divergence between the exogenous ethephon treatment group and the control group, at 0,24 and $48 \mathrm{~h}$, intercalary meristems (within $2 \mathrm{~cm}$ below the node) of the twelfth internode were collected. Three plants were collected from each group. Nine days after treatment, paraffin sections in the longitudinal and vertical planes of stems were prepared from control and treated seedlings.

To quantify the phenotypic variation, three independent samples (15 maize seedlings in each group) from the treatment and control groups were monitored and analyzed. Statistical analysis was performed using Student's $t$-test.

\subsection{RNA isolation and Affymetric GeneChip hybridization}

Total RNA was extracted from the harvested seedlings (mixed sample from elongating internodes of three maize plants) and subjected to different treatments according to the Trizol reagent (Invitrogen, Beijing, China) protocols. GeneChip Maize Genome Arrays were used for differential gene expression analysis. The microarray consisted of 17555 probe sets for about 14850 Zea mays transcripts, representing 13339 genes. The standard wash and doublestain protocols were applied using a fluidics station (Affymetrix GeneChip Fluidics Station 450). The arrays were scanned on a GeneChip scanner (Affymetrix GeneChip Scanner 3000). Each sample included three biological replicates to determine both the reproducibility and quality of the chip hybridization.

The raw data sets (CEL) and the normalized expression data sets have been deposited in the Gene Expression Omnibus (GSE56062) at the National Center for Biotechnology Information (http://www.ncbi.nlm.nih.gov/geo).

\subsection{Microarray data processing and analysis}

First, the scanned arrays were analyzed using Affymetrix GCOS 1.4 software to generate raw data (estimated probe 
intensity values) and saved as CEL files. Next, DNA-Chip Analyzer 2008 software was used to normalize all 15 array sets $^{[38]}$. The Significance Analysis of Microarrays software was used for screening differentially-expressed genes (http://www.dchip.org). A gene was considered to be expressed when it had detection call $\mathrm{P}$ in all three replicates, with its detection $P<0.05$. We used GeneSpring GX 11 software to analyze the correlation coefficients between every pair of arrays, all correlation coefficients were greater than 0.9. Genes showing statistically significant differential expression of transcriptional changes were identified when two conditions were satisfied: (1) more than twofold upregulation or downregulation after ethephon treatment; (2) a change with a $P<0.05$. Negative or positive values represent downward or upward regulation, respectively. All probe sets (CapitalBio Corporation) were subjected to the statistically enriched Gene Ontology analysis using the AgriGO online service $^{[31]}$ (http://bioinfo.cau.edu.cn/agriGO); the hormone signaling pathways associated with the differentially-expressed genes were identified on the basis of a background distribution from the whole chip using Kyoto Encyclopedia of Genes and Genomes (KEGG; version 43) and grouped into functional categories and metabolic pathways using the Molecule Annotation System MAS 3.0 (http://bioinfo.capitalbio.com/mas3) of CapitalBio Corporation. Microsoft Excel and Access were used to extract and manage microarray data.

\subsection{Real-time quantitative reverse transcription polymerase chain reaction analysis}

Total RNA samples from maize internodes for GeneChip experiment (three biological replicates) were used for realtime polymerase chain reaction (PCR) assays in order to ensure the reliability and repeatability of the results. Genomic DNA contamination was eliminated by treating the RNA samples with DNase I (TaKaRa, Dalian, Liaoning, China). Then, the total RNA was used to synthesize cDNA in a reverse transcription reaction using random primers (Promega, Madison, WI, USA). The cDNA samples were diluted to $4 \mathrm{ng} \cdot \mu \mathrm{L}^{-1}$. The genespecific primers (Appendix A, Table S1) were designed using Primer 3 (http://frodo.wi.mit.edu/primer3/input. $\mathrm{htm})$. As a positive control, 18S rRNA was used to normalize the data. Three biological replicates were assayed using the Power SYBR Green PCR Master Mix (Applied Biosystems, Foster City, CA, USA) on a 7500 Real Time PCR System machine (Applied Biosystems) according to the manufacturer's protocols. The amplification reactions were performed under the following PCR conditions: one cycle at $94^{\circ} \mathrm{C}$ for $5 \mathrm{~min}$, followed by 40 cycles of $94^{\circ} \mathrm{C}$ for $30 \mathrm{~s}, 58^{\circ} \mathrm{C}$ for $30 \mathrm{~s}$, and $72^{\circ} \mathrm{C}$ for $45 \mathrm{~s}$, a final extension at $72^{\circ} \mathrm{C}$ for $7 \mathrm{~min}$, and holding at $4^{\circ} \mathrm{C}$. The average $\mathrm{Ct}$ values of three different samples were calculated for different genes and references. The relative changes in expression were calculated using the relative $2^{-\Delta \Delta C_{\mathrm{T}}}$ method $^{[39]}$.

\section{Results}

\subsection{Phenotypic responses to ethephon treatment}

As shown in Fig. 1a and Fig. 1b, the lengths of the eleventh, twelfth and thirteenth internode of the ethephontreated plants were significantly shorter than in the control plants (Appendix A, Table S2). This change in internode elongation of Zong 31 in response to exogenous ethephon treatment was related to the morphology of intercalary meristems cells and cytological observations revealed that ethylene release inhibited the longitudinal growth of cells and promoted lateral growth. The cross section and the longitudinal sections showed that the ethephon-treated internodes had shorter and wider cells, and greater vascular density (Figs. 1c-1g), with the average cell length (Appendix A, Table S3) decreasing from 68.9 to $32.6 \mu \mathrm{m}$, and the average width increased from 92.6 to $103 \mu \mathrm{m}$, respectively.

\subsection{Differentially-expressed genes involved in internode elongation by ethephon treatment}

Ethephon triggered reduced maize internode length probably by decreasing the longitudinal growth of cells. Previous studies have shown consistent results in maize, wheat and barley ${ }^{[3-7]}$. Accordingly, we selected three time points $(0,24$ and $48 \mathrm{~h})$ to investigate the differential expression of genes at the transcription level, and at every point setting, three biological samples were collected independently for analysis. The Affymetrix Zea mays whole genome chip was designed mainly from the representative maize inbred genotypes B73, Ohio 43 and W22. We first tested probe level between the treatments and the control for Zong 31. The average percentage of 17555 probes varied from $72.8 \%$ to $76.1 \%$, indicating that the polymorphic probes will probably not significantly impact our results (Appendix A, Table S4). Of the tested probes, 326 genes showed significant changes in expression in response to ethephon after $24 \mathrm{~h} ; 190$ were significantly upregulated and 136 were significantly downregulated, accounting for $58.3 \%$ and $41.7 \%$ of the total number of differentially-expressed genes, respectively. After $48 \mathrm{~h}$ of treatment, 89 differentially-expressed genes were detected; 55 were significantly upregulated and 34 were significantly downregulated, accounting for $61.8 \%$ and $38.2 \%$ of the differentially-expressed probes, respectively (Table 1). Furthermore, the number of differentially-expressed probes sets identified in the ethephon treatment at $48 \mathrm{~h}$ was smaller than at $24 \mathrm{~h}$. Specifically, most of the differentially-expressed genes were regulated by two to 5-fold, while a few were 
(a)

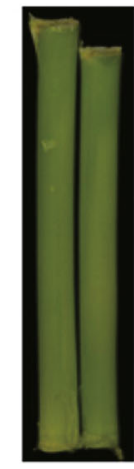

$\mathrm{CK}^{\mathrm{Eth}}{ }^{+}$

Eleventh

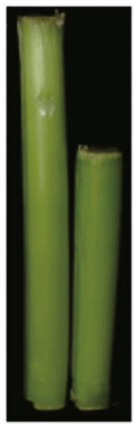

CK Eth ${ }^{+}$

Twelfth

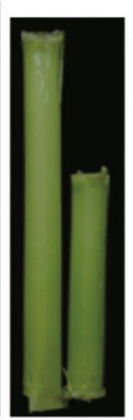

CK Eth ${ }^{+}$ Thirteenth

(c) $50 \mu \mathrm{m}$

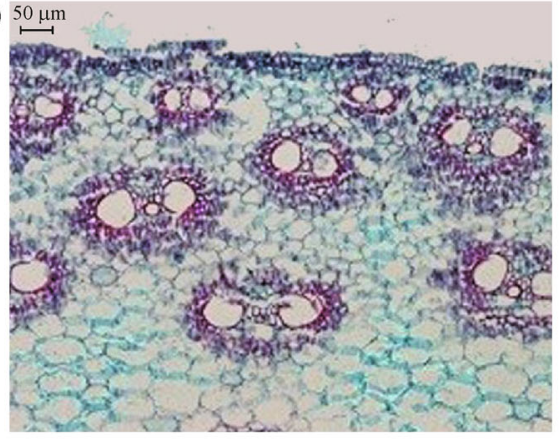

(e)

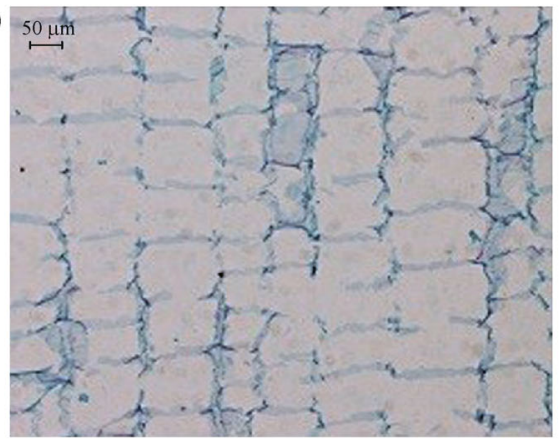

(g)

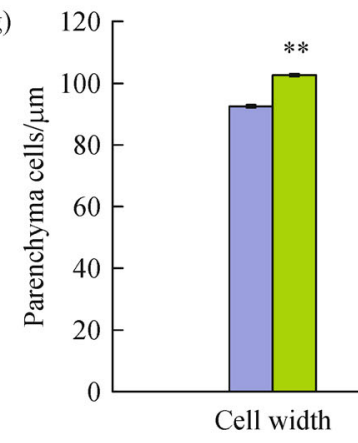

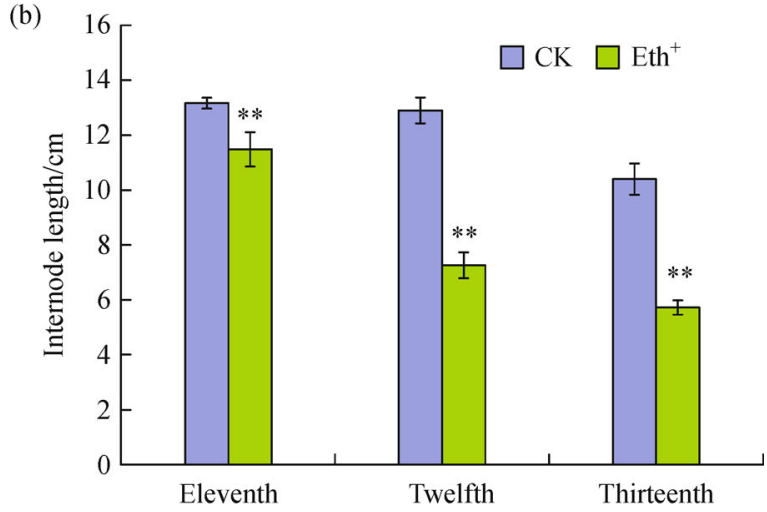

(d) $\underset{\longmapsto}{5} \mu \mathrm{m}$

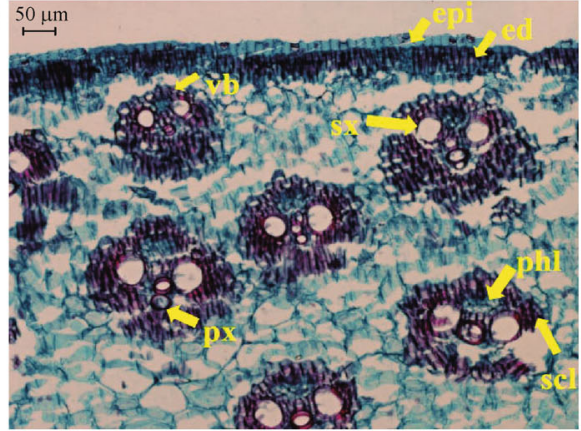

(f)

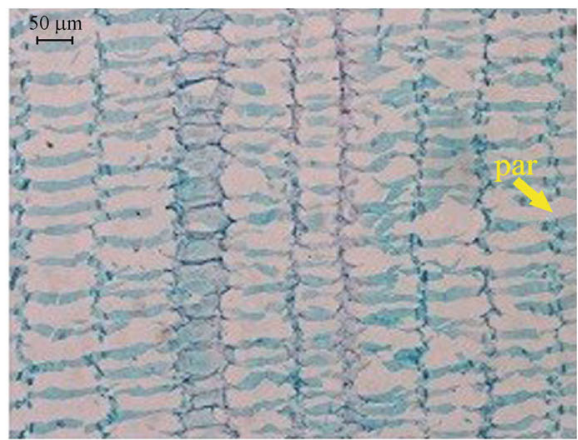

$\square \mathrm{CK} \square \mathrm{Eth}^{+}$

Fig. 1 Morphological characterization $9 \mathrm{~d}$ after ethephon treatment $\left(\mathrm{Eth}^{+}\right)$and control (CK). (a) Phenotypic characterization of the eleventh, twelfth and thirteenth nodes of maize cv. Zong 31 plants; control (CK) and ethephon treatment $\left(E^{+}\right)^{+}$. Scale bar $=2 \mathrm{~cm}$; (b) comparison of the length of the eleventh, twelfth and thirteenth nodes of Zong 31, at least 30 plants were analyzed. Asterisks indicate significant differences between control $(\mathrm{CK})$ and ethephon treatment $\left(\mathrm{Eth}^{+}\right)(* *, P<0.01$; Student's $t$-test). Values indicate mean $\pm \mathrm{SD}$; $(\mathrm{c}-\mathrm{d})$ paraffin-cut cross section $(\times 10)$ of the eighth nodes of Zong 31 at the mature stage after control (c) and ethephon treatment $(\mathrm{d})$. Dark coloration indicates the presence of syringyl lignin units. epi, epidermis; ed, exodermis; vb, vascular bundle; sx, secondary xylem; px, protoxylem; phl, phloem; scl, sclerenchyma. Scale bar $=50 \mu \mathrm{m}$; (e-f) paraffin-cut longitudinal section $(\times 10)$ of eighth nodes of Zong 31 with control (e) and ethephon treatment (f), respectively, at the mature stage. par, parenchyma cell. Scale bar $=50 \mu \mathrm{m} ;(\mathrm{g})$ length and width of parenchyma cells in paraffin-cut longitudinal section (e-f). Three slides of each sample were observed, and more than 100 cells were measured for each slide. Asterisks indicate significant differences between control (CK) and ethephon treatment $\left(\right.$ Eth $\left.{ }^{+}\right)$. Values are means \pm SD $(n>300)$. Student's $t$-test was used to calculate the $P$ value $(* *, P<0.01)$. 
Table 1 Percent of differentially-expressed probe sets with fold change by ethephon treatment

\begin{tabular}{|c|c|c|c|c|c|c|}
\hline \multirow{2}{*}{ Treatment } & \multirow{2}{*}{ Category } & \multirow{2}{*}{ Number of probe sets } & \multicolumn{4}{|c|}{ Fold change $/ \%$} \\
\hline & & & $2-3$ & $3-5$ & $5-10$ & $10-50$ \\
\hline \multirow{2}{*}{$24 \mathrm{~h}$ Eth vs. $24 \mathrm{~h} \mathrm{CK}$} & Upregulated & 190 & 51.05 & 28.95 & 12.11 & 7.89 \\
\hline & Downregulated & 136 & 69.85 & 22.06 & 5.15 & 2.94 \\
\hline \multirow{2}{*}{$48 \mathrm{~h}$ Eth vs. $48 \mathrm{~h} \mathrm{CK}$} & Upregulated & 55 & 50.91 & 38.18 & 7.27 & 3.64 \\
\hline & Downregulated & 34 & 50.00 & 41.18 & 5.88 & 2.94 \\
\hline
\end{tabular}

regulated by five to 10 -fold or even up to 50 -fold, which was similar to the trend observed after $24 \mathrm{~h}$ of treatment. These findings indicate that exogenous ethephon had differentially induced changes in expression of internode genes at differing time points.

To further investigate the similarities between the differentially-expressed genes caused by ethephon at different time points, we performed a hierarchical cluster analysis (Fig. 2a). The genes showing changes in expression at 24 and $48 \mathrm{~h}$ showed similar expression patterns, which were different from those of genes showing changes in expression at $0 \mathrm{~h}$. This suggests that the changes in transcript levels at $0 \mathrm{~h}$ may represent responses of maize internodes to small environmental changes during growth and development. However, the significant differences between 24 and $48 \mathrm{~h}$ were caused by ethephon. Further analysis revealed that the differentially-expressed genes showed greater reproducibility and consistency at $24 \mathrm{~h}$ than at $48 \mathrm{~h}$. This indicates that the maximal effect of ethephon on maize internode elongation occurred within $48 \mathrm{~h}$, and the peak number of differentially-expressed genes occurred at $24 \mathrm{~h}$.

To present the differences between the genes differentially expressed at various time points, we constructed a (a)

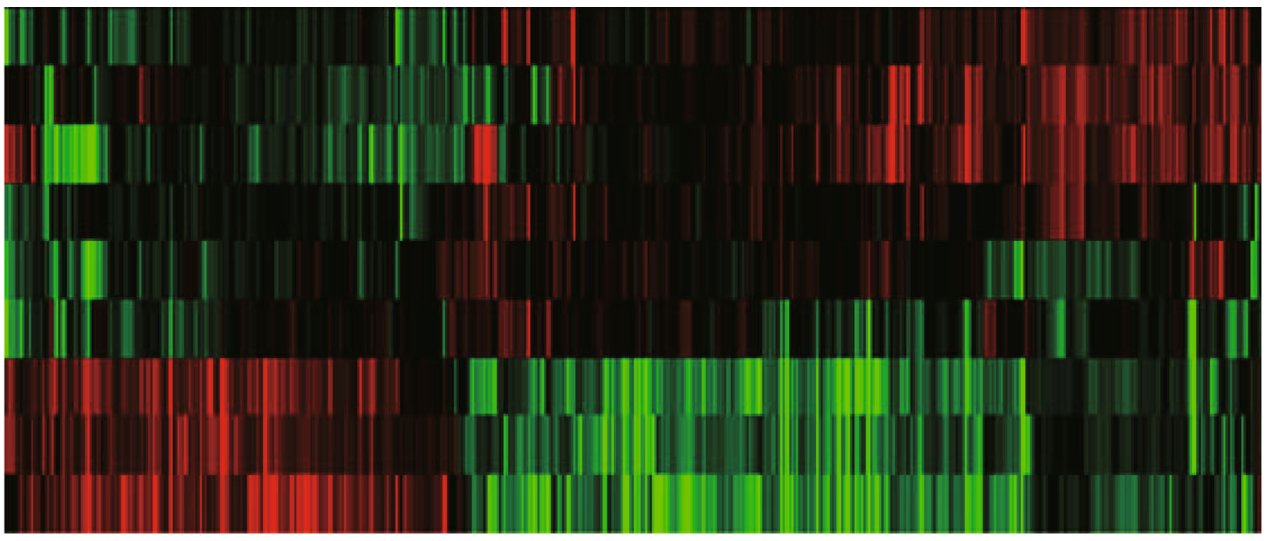

24 Eth I

24 Eth II

24 Eth III

48 Eth I

48 Eth II -3.00

48 Eth III -2.00

0 Eth I

1.00

2.00

0 Eth III $\quad 3.00$ (b)

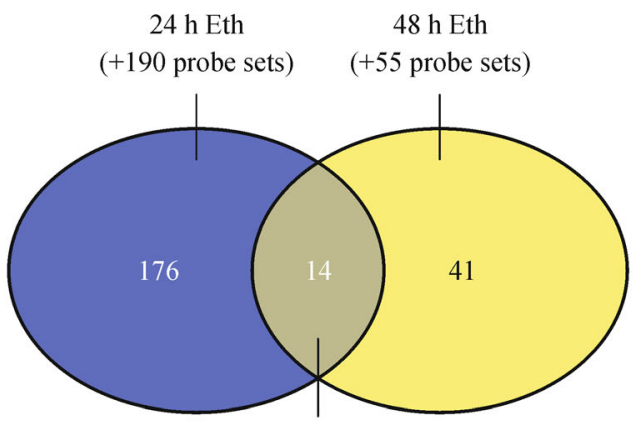

Common

14 probe sets were commonly up-regulated (c)

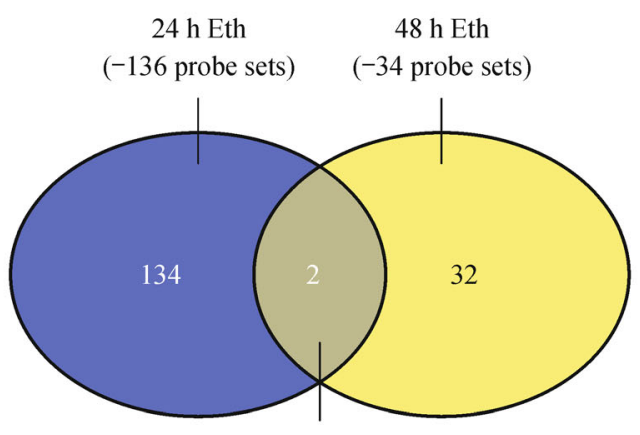

Common

2 probe sets were commonly down-regulated

Fig. 2 Hierarchical cluster analysis and Venn diagrams of differentially-expressed genes in response to ethephon treatment. (a) Hierarchical cluster analysis of differentially-expressed genes in response to ethephon treatment. 0 Eth, ethephon treatment 0 h; 24 Eth, ethephon treatment 24 h; 48 Eth, ethephon treatment 48 h. I, II, III indicate biological replicates; (b) the upregulated probe sets for ethephon treatment after 24 and $48 \mathrm{~h}$; (c) the downregulated probe sets for ethephon treatment after 24 and $48 \mathrm{~h}$. 
Venn diagram (Fig. 2b, Fig. 2c) which showed that, of the upregulated genes, 190 probe sets were detected at $24 \mathrm{~h}$, and 55 probe sets were detected at $48 \mathrm{~h}$. Further analyses revealed that 14 probe sets were sustained and had stable upregulation at 24 and $48 \mathrm{~h}$ (Fig. 2b). Of the downregulated genes, 136 probe sets were detected $24 \mathrm{~h}$, and 34 were detected $48 \mathrm{~h}$. Further analyses showed two probe sets were persistently downregulated at 24 and $48 \mathrm{~h}$ (Fig. 2c). These 16 probe sets were continuously expressed throughout the ethephon treatment, which was consistent with results of the hierarchical cluster analysis, suggesting that these probe sets may be potentially important in Zong 31 internode cell elongation.

\subsection{Gene ontology analysis of the differentially-expressed} genes

GO ontology is widely applied to understand the biological significance of differential gene expression data obtained using microarrays. To evaluate the potential functions of these differentially-expressed genes after $24 \mathrm{~h}$ in response to ethephon, we performed GO category enrichment analysis for 326 probe sets using the AgriGO online service (http://bioinfo.cau.edu.cn/agriGO). In the Affymetrix maize microarray, these 326 genes are represented by 326 probe sets. As shown in Table 2 and Table 3, 75.3\% of 190 upregulated probe sets and $69.9 \%$ of 136 down- regulated probe sets with GO term annotations were identified.

The GO categories (Table 2) and results of enrichment analysis for the 190 upregulated probe sets, which contained 13162 annotated reference items on the whole genome chip, were classified into 16 functional categories. A total of 143 probe sets with GO term annotations were identified $(P<0.05)$. Further analyses revealed that the most significantly enriched GO term of the biological processes was cell wall macromolecule catabolic process (GO:0016998, $8.90 \times 10^{-6}$ ). Other GO terms were also enriched, such as the cell wall macromolecule metabolic process (GO:0044036, 5.70 $\times 10^{-5}$ ), cellular amino acid derivative metabolic processes (GO:0006575, 0.002), and the multi-organism process (GO:0051704, 0.044). In the cellular component, there were 22 probe sets each with GO term annotations for cytoplasmic membrane-bound vesicle (GO:0016023, 0.0018), membrane-bound vesicle (GO:0031988, 0.0018), vesicle (GO:0031982, 0.002), and cytoplasmic vesicle (GO:0031410, 0.002). The functions of these probe sets in plants included gibberellin metabolic process, ethylene binding, ethylene-mediated signaling pathway, electron transport, hydrolase activity, peroxidase activity, lignin biosynthetic process, stilbene biosynthetic process, amino acid metabolic process, oxidoreductase activity and chloroplast binding. For molecular functions, there were nine, nine, seven, six

Table 2 Gene ontology analysis of upregulated probe sets responsive to ethephon treatment

\begin{tabular}{|c|c|c|c|c|}
\hline GO ID & GO term & Query item & Background item & $P$ value \\
\hline \multicolumn{5}{|c|}{ Biological process } \\
\hline GO:0016998 & Cell wall macromolecule catabolic process & 5 & 22 & $8.90 \times 10^{-6}$ \\
\hline GO:0044036 & Cell wall macromolecule metabolic process & 5 & 34 & $5.70 \times 10^{-5}$ \\
\hline GO:0006575 & Cellular amino acid derivative metabolic process & 9 & 251 & 0.0020 \\
\hline GO:0051704 & Multi-organism process & 5 & 174 & 0.0440 \\
\hline \multicolumn{5}{|c|}{ Cellular component } \\
\hline GO:0016023 & Cytoplasmic membrane-bound vesicle & 22 & 1027 & 0.0018 \\
\hline GO:0031988 & Membrane-bound vesicle & 22 & 1027 & 0.0018 \\
\hline GO:0031982 & Vesicle & 22 & 1035 & 0.0020 \\
\hline GO:0031410 & Cytoplasmic vesicle & 22 & 1035 & 0.0020 \\
\hline \multicolumn{5}{|c|}{ Molecular function } \\
\hline GO:0050825 & Ice binding & 9 & 135 & $2.50 \times 10^{-5}$ \\
\hline GO:0050824 & Water binding & 9 & 135 & $2.50 \times 10^{-5}$ \\
\hline GO:0016798 & Hydrolase activity, acting on glycosyl bonds & 7 & 153 & 0.0017 \\
\hline GO:0004553 & Hydrolyzing O-glycosyl compounds & 6 & 130 & 0.0035 \\
\hline GO:0005506 & Iron ion binding & 8 & 264 & 0.0091 \\
\hline GO:0003677 & DNA binding & 15 & 752 & 0.0180 \\
\hline GO:0020037 & Heme binding & 5 & 142 & 0.0210 \\
\hline GO:0046906 & Tetrapyrrole binding & 5 & 157 & 0.0310 \\
\hline Total & & $143(75.3 \%)$ & 13162 & \\
\hline
\end{tabular}


probe sets respectively with $\mathrm{GO}$ term annotations for ice binding (GO:0050825, 2.50 $\times 10^{-5}$ ), water binding (GO:0050824, $2.50 \times 10^{-5}$ ), hydrolase activity, action on glycosyl bonds (GO:0016798, 0.0017) and hydrolyzing Oglycosyl compounds (GO:0004553, 0.0035), respectively. Their functions included cell wall macromolecule catabolic process, cation binding, carbohydrate metabolic process and rRNA N-glycosylase activity. There were 8, 15,5 , and 5 probe sets with GO term annotations for iron ion binding (GO:0005506, 0.0091), DNA binding (GO:0003677, 0.018), heme binding (GO:0020037, 0.021 ), and tetrapyrrole binding (GO:0046906, 0.031), respectively. The functions of these probe sets in plant included iron ion transport, iron ion binding, electron transport, gibberellin metabolic process, peroxidase activity, oxygen transporter activity, oxygen transport, transcription factor activity, regulation of transcription, defense response, ethylene binding, protein histidine kinase, gibberellin metabolic process, heme binding and cytoplasmic membrane-bound vesicle formation.

One hundred and thirty-six probe sets were downregulated according to $\mathrm{GO}$ categories and enrichment analysis, which contained 13162 annotated reference items on the whole genome chip. These were classified into 10 functional categories, including only biological process and cellular component, and a total of 95 probe sets with GO term annotations were identified (Table 3). Further analyses revealed that the most significant enriched GO term was RNA metabolic process (GO:0016070, 0.0015) in biological processes. Also there were other GO terms enriched, including regulation of transcription, DNAdependent (GO:0006355, 0.033), nucleic acid metabolic process (GO:0006139, 0.037), regulation of RNA metabolic process (GO:0051252, 0.044) and regulation of biological process (GO:0050789, 0.049). The functions of these probe sets in plants included signal transduction (phosphorelay), chromatin assembly or disassembly, structural constituent of chromatin, transcription factor activity, DNA topological changes, pyrimidine nucleotide biosynthetic process, mitotic cell cycle spindle assembly checkpoint, cell division, salicylic acid-mediated signaling pathway and jasmonic acid (JA) mediated signaling pathway. For the cellular component, there were 14 probe sets each with GO term annotations for cytoplasmic membrane-bound vesicle (GO:0016023, 0.016), membrane-bound vesicle (GO:0031988, 0.016), vesicle (GO:0031982, 0.017), and cytoplasmic vesicle (GO:0031410, 0.017). The functions of these probe sets in plants include gibberellin metabolic process, ethylene binding, ethylene-mediated signaling pathway, cytoplasmic membrane-bound vesicle, metal ion binding, and electron transport. In addition, 10 probe sets GO terms included chloroplast (GO:0009507, 0.045); their functions in plants include hydrolase activity, unfolded protein binding, embryonic development, chloroplast organization, DNA topological change and response to chlorate.

The results of GO analysis indicated that the differentially-expressed genes were enriched $(P<0.05)$ in biological process, cellular component, and molecular function classifications. Notably, cell wall macromolecule catabolic process (GO:0016998, $8.90 \times 10^{-6}$ ) was significantly enriched among the 190 upregulated probe sets and RNA metabolic process (GO:0016070, 0.0015) was significantly enriched among the 136 downregulated probe sets. Also many genes were predicted to be involved in amino acid metabolic process, lignin biosynthetic process, transcription factor activity, defense response, metal ion binding, electron transport and IAA/GA metabolic processes. Ethylene/jasmonic acid-mediated signaling genes were also preferentially differentially expressed, indicating that various factors in cellular size have a specific function in the maize internode elongation process induced by

Table 3 Gene ontology analysis of down-regulated probe sets responsive to ethephon treatment

\begin{tabular}{llccc}
\hline GO ID & \multicolumn{1}{c}{ GO term } & Query item & Background item & $P$ value \\
\hline Biological process & & & 693 \\
GO:0016070 & RNA metabolic process & 13 & 419 & 0.0015 \\
GO:0006355 & Regulation of transcription, DNA-dependent & 7 & 1373 \\
GO:0006139 & Nucleic acid metabolic process & 16 & 446 \\
GO:0051252 & Regulation of RNA metabolic process & 7 & 1421 \\
GO:0050789 & Regulation of biological process & 16 & 0.0330 \\
Cellular component & & & 0.0370 \\
GO:0016023 & Cytoplasmic membrane-bound vesicle & 14 & 1027 \\
GO:0031988 & Membrane-bound vesicle & 14 & 1027 \\
GO:0031982 & Vesicle & 14 & 1035 \\
GO:0031410 & Cytoplasmic vesicle & 14 & 1035 \\
GO:0009507 & Chloroplast & 10 & 0.0160 \\
Total & & $95(69.9 \%)$ & 0.0160 \\
\hline
\end{tabular}


ethephon. Plant organ growth is a prerequisite for both cell elongation and division ${ }^{[40,41]}$, as well as for maize internode elongation and changes in cell morphology. The elongation and division of the meristem cells is largely regulated by the feedback regulation mechanism of small molecular compounds and biological macromolecules involved in cell metabolism. Overall, the analysis implies that exogenous ethephon treatment may change cell metabolism through a mechanism involving a network of small molecular compounds and biological macromolecules. The functional categories of differentially-expressed genes associated with cell elongation and division by ethephon treatment in maize internodes were further analyzed on the basis of the results of GO term annotations and gene classifications (Table 4 and Table 5).

\subsubsection{Cell and cell wall component synthesis proteins}

Further analysis of the functional categories of differentiallyexpressed genes indicated that six probe sets related to cell and cell wall component synthesis changed significantly. Of these, probe sets Zm.7715.1.A1_at (Wiscott-Aldrich syndrome, C-terminal) and Zm.17188.1.A1_at (Arabinogalactan protein) were upregulated more than 10 -fold, and Zm.16805.3.S1_at (Chitinase) and Zm.2147.1.A1_s_at (Glycine-rich cell wall structural protein) were upregulated more than twofold. Cell and cell wall biogenesis is a complex process involving the action of many protein families directly involved in the synthesis of cell and cell polysaccharides and the rearrangement of polymers ${ }^{[42]}$. $Z m G R P 3^{[43]}$ and $Z m G R P 4^{[44]}$ have been reported to be root specific, with the highest expression level in the meristematic and elongation regions, as putative signal peptides become insoluble within the cell wall. As for two downregulated probe sets, one gene was associated with a cell cycle checkpoint protein MAD2 (Zm.16475.1.S1_at) homolog, which is related to the microtubules of internode cells. The other gene (Zm.6789.1.A1_at) was the cell division cycle protein 20. Furthermore, the cell wall of maize stalks represents a major energy storage compartment as most of the solar energy captured by plants is photosynthetically converted into chemical energy locked into the cell wall polymers: cellulose, hemicellulose, and lignin $^{[42]}$. These are important factors that can directly affect cell elongation and extension, suggesting that ethephon treatment may influence differentially-expressed gene responses affecting internode cell polysaccharides and the rearrangement of polymers.

\subsubsection{Stress-responsive genes}

There were nine probe sets that changed significantly related to the stress response functional category. Of these, five probe sets (Zm.10149.1.A1_at, Zm.94.1.S1_at, Zm.17335.1.S1_at, Zm.2193.1.A1_at and Zm.1449.2.
S1_a_at) were induced strongly during drought stress and were upregulated more than twofold in response to ethephon ${ }^{[29]}$. These are putatively homologs of dehydrin COR410, Dof2, stachyose synthase, glutathione transferase 20 and $\mathrm{NaCl}$ stress protein 1 in Arabidopsis, respectively. The four downregulated probe sets, $\mathrm{Zm}$. 283.1.S1_at included genes involved in production of defensive chemicals, putatively encoding tryptophan synthase and bx 1 (benzoxazinless 1) were downregulated more than 12-fold. The other three probe sets $(Z m .2488 .1$. A1_at, Zm.16643.1.S1_at, and Zm.5799.1.A1_at), which are putative homologs of CTP synthase, peroxidase 27 and gamma-tocopherol methyltransferase, were downregulated two to 5 -fold. These genes, preferentially expressed in response to internal and external stimuli, can trigger a wide range of developmental processes in plants. For example, Zm.5799.1.A1_at upregulation enhances antioxidants in maize ${ }^{[45]}$, implying that these are excellent candidates for involvement in the biosynthetic processes and cellular organization of internodes.

\subsubsection{Transcription factors}

Transcription factors act as master regulators of cellular processes for modifying complex traits in crop plants. Twenty two transcription factor genes were detected and predicted to be excellent candidates for involvement in the response to ethephon. The expression of 10 probe sets was upregulated more than twofold, with Zm.82.1.S1_at (b1) more than 15-fold and Zm.10147.1.A1 at (NAC̄1 transcription factor) and Zm.18537.1.S1_at (leucine zipper protein) more than threefold. The other six probe sets (Zm.217.1.S1_at, Zm.94.1.S1_at, Zm.4529.1.A1_a_at, Zm.12864.1.S1_at, Zm.3709.1.S1_at and Zm.275.1. S1_a_at), which are the putative homologs of MAP kinase $4, \bar{D}$ of 2 , protease inhibitor, nuclear transcription factor, $R N A$ recognition motif containing protein and ethylene receptor 2, were upregulated twofold. Among the 12 downregulated probe sets, five probe sets (Zm.9708.1. A1_at, Zm.325.1.A1_at, Zm.14450.1.A1_at, Zm.4871.1. $A 1 \_a t$ and Zm.6485.1.A1_at) were downregulated three to 5-fold, including the putative homologs of bhlh84-DNA binding protein, RR6-response regulator, SBP-domain protein 5, growth-regulating factor 1, general regulatory factor 2. The other seven probe sets (Zm.1066.3.A1_at, Zm.6732.3.A1_at, Zm.19219.1.A1_at, Zm.326.1.S1_at, Zm.5439.1.S1_at, Zm.11846.1.A1_at, Zm.289.1.S1_at), which are the putative homologs of $S B P$-domain containing protein, HMG1/2-like protein, RNA binding protein, $R R 7$, serine/threonine-protein kinase 12, SBP-domain protein 7, Rop6 protein, respectively, were downregulated twofold. In previous studies, most attention was directed to the MYB and NAC transcription factors, however, transcription factors belonging to other families might also be involved in regulating cell wall biogenesis. In this 
Table 4 Differentially-expressed cell component, stress-responsive, and transcription factor genes in the internode elongation response to ethephon treatment

\begin{tabular}{|c|c|c|c|}
\hline Probe set ID & Changing fold & Gene ID & Description \\
\hline \multicolumn{4}{|c|}{ Cell and cell wall component synthesis proteins } \\
\hline Zm.7715.1.A1_at & 18.16 & GRMZM2G001661 & Wiscott-Aldrich syndrome protein, C-terminal \\
\hline Zm.17188.1.A1_at & 9.87 & GRMZM2G043035 & Polypeptide: Arabinogalactan protein \\
\hline Zm.16805.3.S1_at & 4.50 & GRMZM2G099454 & Enzyme: Chitinase \\
\hline Zm.2147.1.A1_s_at & 2.68 & GRMZM2G109959 & Glycine-rich cell wall structural protein \\
\hline Zm.16475.1.S1_at & 0.33 & GRMZM2G047143 & Cell cycle checkpoint protein MAD2 \\
\hline Zm.6789.1.A1_at & 0.27 & GRMZM2G063192 & Cell division cycle protein 20 \\
\hline \multicolumn{4}{|c|}{ Stress-responsive genes } \\
\hline Zm.10149.1.A1_at & 2.77 & GRMZM2G147014 & Dehydrin COR410 \\
\hline Zm.94.1.S1_at & 2.49 & GRMZM2G009406 & Dof 2 (DNA binding with one finger 2) \\
\hline Zm.17335.1.S1_at & 2.42 & GRMZM2G340656 & Stachyose synthase \\
\hline Zm.2193.1.A1_at & 2.29 & GRMZM2G434541 & Glutathione transferase 20 \\
\hline Zm.1449.2.S1_a_at & 2.00 & GRMZM2G015605 & $\mathrm{NAC} 1(\mathrm{NaCl}$ stress protein 1$)$ \\
\hline Zm.2488.1.A1_at & 0.43 & GRMZM2G153058 & CTP synthase family protein \\
\hline Zm.16643.1.S1_at & 0.36 & GRMZM2G320269 & Peroxidase 27 (peroxidase superfamily protein) \\
\hline Zm.5799.1.A1_at & 0.31 & GRMZM2G035213 & Gamma-tocopherol methyl transferase \\
\hline Zm.283.1.S1_at & 0.08 & GRMZM2G085381 & bx1 (benzoxazinless1, tryptophan synthase) \\
\hline \multicolumn{4}{|l|}{ Transcription factors } \\
\hline Zm.82.1.S1_at & 15.07 & GRMZM2G172795 & b1 (colored plant1, DNA binding domain) \\
\hline Zm.10147.1.A1_at & 6.80 & GRMZM2G347043 & NAC1 transcription factor(nactf49) \\
\hline Zm.18537.1.S1_at & 5.95 & GRMZM2G117164 & homeobox-leucine zipper protein ATHB-6 \\
\hline Zm.10253.1.A1_at & 3.88 & GRMZM2G134334 & Multiple stress-responsive zinc-finger protein ISAP1 \\
\hline Zm.217.1.S1_at & 2.75 & GRMZM2G127141 & MAP kinase 4 \\
\hline Zm.94.1.S1_at & 2.49 & GRMZM2G009406 & Dof 2 (DNA binding with one finger 2) \\
\hline Zm.4529.1.A1_a_at & 2.17 & GRMZM2G065557 & Protease inhibitor/seed storage/LTP family \\
\hline Zm.12864.1.S1_at & 2.12 & GRMZM5G857944 & Nuclear transcription factor Y subunit A-3 \\
\hline Zm.3709.1.S1_at & 2.08 & GRMZM2G114325 & RNA recognition motif containing protein \\
\hline Zm.275.1.S1_a_at & 2.07 & GRMZM2G420801 & Ethylene receptor homolog (etr2,Histidine kinase) \\
\hline Zm.1066.3.A1_at & 0.48 & GRMZM2G067624 & SBP-domain containing protein (sbp29) \\
\hline Zm.6732.3.A1_at & 0.47 & GRMZM2G162284 & HMG1/2-like protein(high mobility group) \\
\hline Zm.19219.1.A1_at & 0.45 & GRMZM2G134398 & RNA binding protein (NusB family) \\
\hline Zm.326.1.S1_at & 0.43 & GRMZM2G096171 & RR7 (response regulator 7) \\
\hline Zm.5439.1.S1_at & 0.43 & GRMZM2G107689 & serine/threonine-protein kinase 12 \\
\hline Zm.11846.1.A1_at & 0.40 & GRMZM2G460544 & SBP-domain protein 7 \\
\hline Zm.289.1.S1_at & 0.40 & GRMZM2G176217 & Rop6 protein (rhoptry protein) \\
\hline Zm.6485.1.A1_at & 0.32 & GRMZM2G078641 & General regulatory factor2 (14-3-3 protein) \\
\hline Zm.4871.1.A1_at & 0.30 & GRMZM2G178261 & Growth-regulating factor 1 \\
\hline Zm.14450.1.A1_at & 0.28 & GRMZM2G160917 & SBP-domain protein 5 \\
\hline Zm.325.1.A1_at & 0.26 & GRMZM2G040736 & RR6 (type-A response regulator) \\
\hline Zm.9708.1.A1_at & 0.17 & GRMZM2G159456 & bhlh84, DNA binding protein \\
\hline
\end{tabular}

Note: Changes $\geqslant 2$ fold indicate differentially upregulated genes; Change $\leqslant 0.5$ fold indicated differentially downregulated genes.

study, ethylene receptor 2 , SBP-domain containing protein were shown to up or downregulate the internode develop- ment process involved in cell growth following ethephon treatment, indicating that some of these regulatory 
Table 5 Differentially-expressed transporter, metabolic pathway, plant hormone synthesis and signal transduction genes in response to ethephon treatment

\begin{tabular}{|c|c|c|c|c|}
\hline Probe Set ID & Changing fold & Gene ID & Description & Pathway \\
\hline \multicolumn{5}{|l|}{ Transporters } \\
\hline Zm.6759.1.A1_at & 10.85 & GRMZM2G472167 & Peptide transporter PTR2 & Oligopeptide transport \\
\hline Zm.9753.1.A1_at & 4.54 & GRMZM2G318213 & Grx_C15-glutaredoxin subgroup III & Electron transport \\
\hline Zm.390.1.S1_at & 3.30 & GRMZM2G093195 & d3 (dwarf plant 3 , cytochrome $\mathrm{P} 450$ protein) & Electron transport \\
\hline Zm.16578.1.A1_s_at & 3.22 & GRMZM2G126582 & Aquaporin NIP-type & Transport \\
\hline Zm.485.1.A1_at & 2.60 & GRMZM2G067402 & Non-symbiotic hemoglobin & Oxygen transport \\
\hline Zm.714.1.A1_at & 2.60 & GRMZM2G010868 & $\begin{array}{l}\text { PLT2 (phospholipid transfer protein } \\
\text { homolog 2) }\end{array}$ & Lipid transport \\
\hline Zm.4529.1.A1_a_at & 2.17 & GRMZM2G065557 & Lipid binding protein & Lipid transport \\
\hline Zm.13789.1.A1_at & 2.10 & GRMZM2G403007 & Nonspecific lipid-transfer protein & Lipid transport \\
\hline Zm.171.1.A2_at & 0.41 & GRMZM2G155021 & Eukaryotic porin & Anion transport \\
\hline Zm.4845.1.S1_at & 0.18 & GRMZM2G131525 & Peroxidase (syntaxin-related protein) & Intracellular protein transport \\
\hline \multicolumn{5}{|c|}{ Metabolic pathway related gene } \\
\hline Zm.722.1.A1_at & 21.51 & GRMZM2G422750 & c2 (colorless2, chalcone synthase) & Chalcone biosynthesis \\
\hline Zm.354.1.S1_at & 6.78 & GRMZM2G114642 & gl1 (glossy1, phospholipid transfer protein) & Metabolism \\
\hline Zm.2869.1.A1_at & 3.88 & GRMZM2G093175 & Asparagine synthetase & Asparagine biosynthesis \\
\hline Zm.17335.1.S1_at & 2.42 & GRMZM2G340656 & Stachyose synthase & Carbohydrate metabolism \\
\hline Zm.17569.1.S1_at & 2.31 & GRMZM2G106263 & Hydroxymethylglutaryl-CoA synthase & Steroid biosynthesis \\
\hline Zm.13251.1.A1_at & 0.49 & GRMZM2G138060 & su1 (sugary1, isoamylase) & Carbohydrate metabolism \\
\hline Zm.17147.1.A1_at & 0.47 & GRMZM2G068506 & Glucose-1-phosphate adenylyltransferase & Glycogen biosynthesis \\
\hline Zm.2488.1.A1_at & 0.43 & GRMZM2G153058 & CTP synthase & Pyrimidine nucleotide metabolism \\
\hline Zm.5799.1.A1_at & 0.31 & GRMZM2G035213 & Gamma-tocopherol methyltransferase & Vitamin E biosynthesis \\
\hline Zm.283.1.S1_at & 0.08 & GRMZM2G085381 & bx1 (benzoxazinless 1, tryptophan synthase) & $\begin{array}{l}\text { Aromatic biosynthesis, } \\
\text { Tryptophan biosynthesis }\end{array}$ \\
\hline \multicolumn{5}{|c|}{ Plant hormone synthesis and signal transduction gene } \\
\hline Zm.722.1.A1_at & 21.51 & GRMZM2G422750 & c2 (colorless 2, chalcone synthase) & IAA (indole-3-acetic acid) \\
\hline Zm.11809.1.A1_at & 13.84 & GRMZM2G380432 & Phosphate-responsive 1 family protein & ABA (abscisic acid) \\
\hline Zm.10147.1.A1_at & 6.80 & GRMZM2G347043 & NAC1 transcription factor (nactf49) & IAA \\
\hline Zm.7548.1.A1_at & 5.35 & GRMZM2G087192 & Opr5 (12-oxo-phytodienoic acid reductase 5) & JA (jasmonic acid) \\
\hline Zm.19276.1.A1_at & 4.92 & GRMZM2G315321 & $\begin{array}{l}\text { Ethylene-responsive element binding } \\
\text { protein } 2\end{array}$ & Ethylene \\
\hline Zm.463.1.A1_at & 3.66 & GRMZM2G014392 & $\begin{array}{l}\text { vp14 (viviparous } 14 \text {, retinal pigment } \\
\text { epithelial membrane protein) }\end{array}$ & $\mathrm{ABA}$ \\
\hline Zm.13480.1.S1_at & 3.57 & GRMZM2G060940 & GA20ox (gibberellin 20 oxidase) & GA (gibberellin) \\
\hline Zm.390.1.S1_at & 3.30 & GRMZM2G093195 & d3 (dwarf plant 3, cytochrome P450 protein) & GA \\
\hline Zm.217.1.S1_at & 2.75 & GRMZM2G127141 & MAP kinase 4 & JA, IAA \\
\hline Zm.275.1.S1_a_at & 2.07 & GRMZM2G420801 & $\begin{array}{l}\text { Ethylene receptor homolog (etr2, } \\
\text { Histidine kinase) }\end{array}$ & Ethylene \\
\hline Zm.5439.1.S1_at & 0.43 & GRMZM2G107689 & serine/threonine-protein kinase 12 & IAA \\
\hline Zm.326.1.S1_at & 0.43 & GRMZM2G096171 & RR7 (response regulator 7) & CTK (cytokinin) \\
\hline Zm.12687.1.S1_a_at & 0.40 & GRMZM2G171702 & Auxin efflux carrier component $1 \mathrm{c}$ & IAA \\
\hline Zm.6485.1.A1_at & 0.32 & GRMZM2G078641 & General regulatory factor 2 (14-3-3 protein) & GA \\
\hline Zm.4871.1.A1_at & 0.30 & GRMZM2G178261 & Growth-regulating factor 1 & GA \\
\hline Zm.325.1.A1_at & 0.26 & GRMZM2G040736 & RR6 (type-A response regulator) & CTK \\
\hline Zm.283.1.S1_at & 0.08 & GRMZM2G085381 & bx1 (benzoxazinless1, tryptophan synthase) & IAA \\
\hline
\end{tabular}

Note: Changes $\geqslant 2$ fold indicate differentially upregulated genes; Change $\leqslant 0.5$ fold indicate differentially downregulated genes. 
functions might well be fulfilled by some of the other transcription factor family members.

\subsubsection{Transporters}

Transporters are essential proteins for plants in various cellular processes, involved in enzyme function, cell elongation, signal transduction and maintenance of the plasma membrane potential ${ }^{[46]}$. Ten probe sets in in the transporters functional category changed significantly. Of these, eight probe sets were upregulated more than twofold and Zm.6759.1.A1 at (Peptide transporter 2) was upregulated more than 10 -fold. The other seven probe sets (Zm.9753.1.A1_at, Zm.390.1.S1_at, Zm.16578.1.A1_s_at, Zm.485.1.A1 at, Zm.714.1.A1 at, Zm.4529.1.A1_a at and $Z m .13789 .1 . A 1$ at) were upregulated two to 5-fold, and were the putative homologs of glutaredoxin subgroup III, d3, aquaporin NIP-type, non-symbiotic hemoglobin, PLT2, Lipid binding protein and nonspecific lipid-transfer protein. Downregulated probe sets (Zm.171.1.A2_at and Zm.4845.1.S1_at), which are the putative homologs of eukaryotic porin and peroxidase, respectively. Ion transport proteins regulate the ion balance inside and outside of cells; lipid transport proteins transfer phospholipids across membranes; water channel proteins remarkably increased the membrane water permeability. Transporter genes exist in all tissues of maize, and the expression of 11 probe sets changed during the internode development process after ethephon treatment, indicating that some of these regulatory functions may also involve in cell elongation and division in maize.

\subsubsection{Metabolic pathway genes}

Ten probe sets in the metabolic pathways functional category changed significantly, and the results of KEGG analysis were consistent with GO classification. Of these, five genes were upregulated more than twofold. $\mathrm{Zm}$.722.1. A1_at (c2), which putatively encodes chalcone synthase, was upregulated more than 20 -fold. The other four probe sets (Zm.354.1.S1 at, Zm.2869.1.A1 at, Zm.17335.1. S1_at and Zm.17569.1.S1_at) were upregulated two to 6fold, and are the putative homologs of phospholipid transfer protein, asparagine synthetase, stachyose synthase, hydroxymethylglutaryl-CoA synthase. Four downregulated probe sets (Zm.17147.1.A1_at, Zm.2488.1.A1at, Zm.5799.1.A1 at, Zm.283.1.S1 at) encode glucose-1-phosphate adenylyltransferase, $\bar{C} T P$ synthase, gamma-tocopherol methyltransferase and tryptophan synthase, respectively. These findings indicate that changes in metabolic pathways are interrelated. The significantly upregulated chalcone synthase may be affected by the uptake and translocation activities of other transporters in maize, which can facilitate the homeostasis of nutrient ions in plant cells. However, further research is required to clarify the details of this regulatory mechanism.

3.3.6 Plant hormone synthesis and signal transduction genes

Plant hormones regulate a wide range of development processes, including seed germination, leaf expansion, stem elongation, flowering, and fruit and seed development ${ }^{[47]}$. Among the functional categories of differentiallyexpressed genes, 17 probe sets were involved in plant hormone synthesis and signal transduction (Table 5). Of these, 10 were upregulated more than twofold. $Z m$.722.1. A1_at (c2), which encodes ochalcone synthase, was upregulated more than 20-fold. Zm.11809.1.A1_at, which encodes a phosphate-responsive protein, was upregulated more than 13-fold. Two probe sets (Zm.10147.1.A1_at, $Z m$.7548.1.A1_at) that were upregulated by 5-fold are the putative homologs of NAC1 transcription factor and Opr5, respectively. Six probe sets (Zm.19276.1.A1 at, Zm.463.1. A1_at, Zm.13480.1.S1_at, Zm.390.1.S1_at, Zm.217.1. S1_at and Zm.275.1.S1_a_at) that were upregulated two to 5 -fold, are the putative homologs of ethylene-responsive element binding protein 2, vp14, GA20ox, d3, MAP kinase 4 and ethylene receptor homolog, respectively. Seven probe sets (Zm.5439.1.S1 at, Zm.326.1.S1 at, Zm.12687. 1.S1_a_at,Zm.6485.1.A1_at,Zm.4871.1.A1_at, Zm.325.1. A1_at, Zm.283.1.S1_at) were downregulated two to 5-fold and putatively encode serine/threonine-protein kinase 12 , $R R 7$, auxin efflux carrier component, general regulatory factor 2, growth-regulating factor 1, RR6 and bxl, respectively. Further analyzed show that six probe sets (Zm.722.1.A1_at, Zm.10147.1.A1_at, Zm.217.1.S1_at, Zm.5439.1.S1_at, Zm.12687.1.S1_a_at and Zm.283.1. A1_at) were involved in IAA synthesis, four probe sets (Zm.13480.1.S1_at, Zm.390.1.S1_at, Zm.6485.1.A1_at and $Z m .4871 .1 . \overline{A 1} a t)$ were related to GA signal transduction, two probe sets (Zm.19276.1.A1_at and Zm.275.1. S1_a_at) were related to ethylene synthesis and signal transduction; two probe sets (Zm.11809.1.A1 at and Zm.463.1.A1_at) were related to ABA signal transduction. The results from previous studies of dwarf mutants indicated that plant hormones, such as GAs, IAA, are important for the control of plant height ${ }^{[48]}$. The microarray data show that Gas and IAA synthesis and signal transduction genes were markedly up or downregulated by ethephon treatment, suggesting that these genes may be important in the internode elongation response to ethylene released in maize plants from ethephon. The findings for genes related to ABA, cytokinin and jasmonic acid imply that crosstalk between ethylene and other plant hormones occur concurrently and closely regulates the internode elongation process in maize, and that exogenous ethephon 
application might cause changes in hormones that inhibits internode elongation.

\subsection{Verification of microarray data by quantitative real-time PCR}

We conducted real-time PCR analysis for 16 randomly selected genes to confirm the validity of the microarray data. A set of 16 differentially-expressed genes were chosen, representing three types of response observed in the microarray analysis: upregulation, downregulation and no expression in control or ethephon treatments. 18S rRNA was used as a reference with a steady level of expression (Primers used are shown in Appendix A, Table S1).

The quantitative real-time PCR results showed that the expression trends of these genes were largely consistent with those of the microarray analysis. However, given the speed of maize internode absorption of released ethylene and the greater sensitivity of real-time $\mathrm{PCR}^{[49-51]}$, the variation of differentially-expressed genes in real-time PCR was considered more significant than those in the microarray analysis (Table 6). For some genes, quantitative real-time PCR suggested a larger difference in expression levels. For example, the transcript encoding a putative Opr5 (12-oxo-phytodienoic acid reductase5) showed a 5.35-fold upregulation $24 \mathrm{~h}$ after ethephon treatment in the microarray analysis ( $\mathrm{Zm}$.7548.1.A1_at), but the quantitative real-time PCR result showed $\overline{64} .3$-fold difference at the same time.

\section{Discussion}

4.1 Internode phenotypes showed significant difference in response to ethephon treatment

We characterized the phenotypic differences in plants of maize inbred line Zong 31 with and without exogenous ethephon treatment (Fig. 1a, Fig. 1b), and the results were consistent with previous research ${ }^{[3,6,23]}$. The characterization of cell morphology by analysis of paraffin sections from the two group revealed that the internode cells of the ethephon treated group had a higher content of syringyl lignin units, and thinner and relatively wider parenchymatous tissue cells than the control group (Fig. 1e, Fig. 1f, and Fig. 1g), which validated the substantial differences between internode elongation in the control and ethephon treated maize.

4.2 Timing of differentially-expressed genes in response to ethephon treatment

All plant growth regulator processes are dynamic. In our results, with the passage of time, the number and intensity of the differentially-expressed genes $48 \mathrm{~h}$ after ethephon treatment might become reduced, which could result from a relatively lower amounts of ethylene released at the cellular level compared to that at $24 \mathrm{~h}$, and thus give rise to a more limited effect on differential expression of transcriptional response (Table 1, Fig. 2a). In the Venn diagram (Fig. 2b, Fig. 2c), a relatively small number of

Table 6 Verification of microarray results by qPCR

\begin{tabular}{|c|c|c|c|c|c|c|}
\hline \multirow{2}{*}{ Probe set ID } & \multirow{2}{*}{ Gene ID } & \multirow{2}{*}{ Description } & \multicolumn{2}{|c|}{ GeneChip } & \multicolumn{2}{|c|}{ Qpcr } \\
\hline & & & $24 \mathrm{~h}$ & $48 \mathrm{~h}$ & $24 \mathrm{~h}$ & $48 \mathrm{~h}$ \\
\hline Zm.217.1.S1_at & GRMZM2G127141 & MAP kinase 4 & 2.75 & 3.16 & 3.81 & 9.22 \\
\hline Zm.82.1.S1_at & GRMZM2G172795 & b1 (colored plant1, DNA binding domain) & 15.07 & 2.84 & 85.12 & 12.84 \\
\hline Zm.10147.1.A1_at & GRMZM2G347043 & NAC1 transcription factor (nactf49) & 6.80 & 4.54 & 21.83 & 18.17 \\
\hline Zm.7548.1.A1_at & GRMZM2G087192 & Opr5 (12-oxo-phytodienoic acid reductase 5) & 5.35 & 3.18 & 64.28 & 5.33 \\
\hline Zm.275.1.S1_a_at & GRMZM2G420801 & Ethylene receptor homolog (etr2, histidine kinase) & 2.07 & 1.43 & 6.50 & 2.93 \\
\hline Zm.11809.1.A1_at & GRMZM2G380432 & Phosphate-responsive 1 family protein & 13.84 & 7.84 & 2.94 & 7.01 \\
\hline Zm.17569.1.S1_at & GRMZM2G106263 & Hydroxymethylglutaryl-CoA synthase & 2.31 & 1.91 & 1.01 & 1.18 \\
\hline Zm.390.1.S1_at & GRMZM2G093195 & d3 (dwarf plant 3 , cytochrome $\mathrm{P} 450$ protein) & 3.30 & - & 8.07 & 4.37 \\
\hline Zm.2147.1.A1_s_at & GRMZM2G109959 & Glycine-rich cell wall structural protein & 2.68 & - & 15.94 & 1.07 \\
\hline Zm.16643.1.S1_at & GRMZM2G320269 & Peroxidase 27 (peroxidase superfamily protein) & 0.36 & 0.74 & 1.31 & 2.99 \\
\hline Zm.326.1.S1_at & GRMZM2G096171 & RR7 (response regulator 7) & 0.43 & 0.72 & 0.17 & 0.18 \\
\hline Zm.171.1.A2_at & GRMZM2G155021 & Eukaryotic porin & 0.41 & 0.89 & 0.88 & 0.94 \\
\hline Zm.12687.1.S1_a_at & GRMZM2G171702 & Auxin efflux carrier component $1 \mathrm{c}$ & 0.40 & 0.62 & 0.15 & 0.21 \\
\hline Zm.16475.1.S1_at & GRMZM2G047143 & Cell cycle checkpoint protein MAD 2 & 0.33 & 0.50 & 0.64 & 0.94 \\
\hline Zm.325.1.A1_at & GRMZM2G040736 & RR6 (type-A response regulator) & 0.26 & 1.17 & 0.19 & 0.87 \\
\hline Zm.283.1.S1_at & GRMZM2G085381 & bx1 (benzoxazinless 1, tryptophan synthase) & 0.08 & 1.08 & 0.11 & 0.52 \\
\hline
\end{tabular}

Note: Dashes indicate undetected. Data in bold represents significant difference at $P<0.01$ in the consecutive comparison of time points. 
probe sets (16) were regulated in common between 24 and $48 \mathrm{~h}$. Thus, with the decrease of ethylene released, the intensity of expression of a few probe sets remained steady and level in a certain time effects (Fig. 2b, Fig. 2c). Therefore, it can be speculated that the probe sets regulated in common were absolutely necessary for response of maize internode elongation to ethephon treatment.

4.3 Internode cell elongation and expression of related differentially-expressed genes in response to ethephon treatment

Internode number and length are two crucial factors affecting plant height. In maize, internode elongation is attributed to the cell division and elongation of intercalary meristems at the base of growing internodes ${ }^{[36,37]}$. As maize stems develop, sclerenchyma and the rind region parenchyma tissues develop thick, lignified cell walls ${ }^{[52]}$. Genes involved in cell and cell wall-related processes might also function in cell growth and cell wall remodeling during a defense response ${ }^{[53]}$. Our results show that the differentially-expressed genes significantly change cell and cell wall component synthesis, transporters, stress-responsive and metabolic pathway genes, and transcription factors.

The functional category, cell and cell wall component synthesis proteins, contained 6 differentially-expressed genes, a probe set $(Z m .7715 .1 . A 1$ at $)$ that putatively encode the homologs of Wiscott-Aldrich syndrome proteins was significantly upregulated, which sends a signal to the cytoskeleton through the Arp2/3 complex, an actinnucleating assembly that regulates the structure and dynamics of actin filament networks at the leading edge of a cell ${ }^{[54]}$. Zm.6789.1.A1 at, which ecodes a putative cell division cycle protein 20 related to cell division, was significantly downregulated; another probe set $(\mathrm{Zm}$. 17188.1.A1_at) encodes a putative arabinogalactan protein which was significantly upregulated, and which is also involved in cell division during pollen germination and pollen tube growth ${ }^{[55]}$. In indica rice, a homolog of an arabinogalactan protein gene OSIAGP, performs a similar function to ATAGP23 in Arabidopsis, and regulates pollen tube growth in the dark ${ }^{[56]}$. Zm.2147.1.A1_s_at encodes a putative glycine-rich cell wall structural protein involved in cell wall component synthesis. It is noteworthy that a similar expression trend in response to ethephon treatment was obtained by quantitative real-time PCR, which showed that Zm.2147.1.A1_s_at was upregulated 2.68-fold in the microarray data. Previous studies have shown that $Z m G R P$ has a putative RNA binding motif ${ }^{[57]}$, and is expressed in the epidermal cells of embryo, scutellar tissue and young leaf, and is induced by ABA, water stress and wounding in leaves ${ }^{[58]}$. The expression of a related gene, $Z m G R P 3$, was root-specific, with the highest expression level in the meristematic and elongation regions ${ }^{[43]}$. Based on these analyses, we confidently hypothesize that $\mathrm{Zm}$.2147.1.
A1_s_at may have a similar mode of action in the intercalary meristem of maize internodes. Zm.16475.1. S1_at was the putative homolog of a cell cycle checkpoint protein MAD2. Previous studies showed that there is a link between MAD2 and cell cycle regulatory proteins in the initiation of anaphase ${ }^{[59]}$. Combined immunolocalization of MAD2 and a recently cloned maize CENPC homolog indicates that MAD2 localizes to an outer domain of the prometaphase kinetochore, and MAD2 staining show that microtubule attachment has a major role in the mitotic spindle checkpoint but the meiotic spindle checkpoint may rely more heavily on sensing the amount of tension at the kinetochore ${ }^{[60]}$. Our study found that Zm.16475.1.S1_at was downregulated 0.33 -fold in quantitative real-time PCR which also agreed with the statistical analysis of microarray data, suggesting that cell cycle checkpoint protein MAD2 might potentially function in maize internode elongation in responses to ethephon treatment ${ }^{[59-62]}$.

In crops, internode cell elongation responds continuously to various biotic and abiotic stresses, which affect the growth of individual plants and the final crop production. With the exception of cell and cell wall component synthesis proteins, the GeneChip results showed that stress-responsive genes, transcription factor, transporters, metabolic pathway-related genes, plant hormone synthesis and signal transduction genes also participate in internode cell elongation process in responses to ethephon treatment. Nine probe sets with stress-responsive genes changed significantly. Among them, Zm.283.1.S1_at, representing the putative homolog of the maize $b x 1$, which catalyzes the conversion of indole-3-glycerol phosphate to indole and also serves as the penultimate intermediate in the formation of tryptophan by tryptophan synthase in secondary metabolism, was downregulated 0.08 -fold ${ }^{[63]}$. Additionally, 22 annotated transcription factor genes were detected (Table 4), suggesting that various transcriptional regulatory mechanisms play important functions in responses to ethephon treatment in intercalary meristems of maize internodes. Among the total differentially-regulated genes were 10 probe sets that were annotated as transporters which changed significantly, which suggests that various regulatory pathways are involved in the cell elongation and cell division responses to ethephon treatment in maize internodes. For example, Zm.6759.1.A1_at, which represents the putative homolog of the peptide transporter PTR2 in Arabidopsis, was upregulated 10.9-fold, and was identified as a flowering and seed development related gene $^{[64]}$. Recent reports show that the homolog gene of the peptide transporter AtPTR1 was localized to the plasma membrane and was shown to expressed in vascular tissue, and to mediate transport of the dipeptide phytotoxin phaseolotoxin ${ }^{[65]}$. In addition, it has been reported that salicylic acid and methyl jasmonate can regulate the expression of AtPTR3 and the mutant has increased susceptibility to virulent bacterial pathogens and contains elevated levels of reactive oxygen species ${ }^{[66]}$. According to 
microarray data, 10 of the sequences homologous to probe sets that changed significantly were putatively participated in metabolic pathways, which suggested that various regulatory metabolic and biosynthesis pathways change in responses to ethephon treatment in maize internodes. For example, $c 2$ ( $\mathrm{Zm}$.722.1.A1_at), representing the putative homolog of the chalcone synthase gene, was significantly upregulated 21.5-fold. Chalcone synthase, the first enzyme in the pathway to flavonoids and anthocyanins, is upregulated that could lead to increased auxin transport ${ }^{[67]}$. This suggests that flavonoids might act as endogenous auxin transport inhibitors in plants ${ }^{[68]}$. Additionally, previous studies have revealed that chalcone synthase was identified as active in almost all maize tissues depending on the expression of different regulatory genes ${ }^{[69]}$, including the pericarp, the aleurone layer of the endosperm tassels ${ }^{[70]}$, and vegetative organs such as ear husks and leaf sheaths ${ }^{[71]}$. Based on the above analysis, phenotypic change in internodes in response to ethephon treatment may result from the interactive effect of these significantly changed genes in intercalary meristem cell level.

4.4 Possible roles of plant hormone pathway and expression in internode cell elongation responses to ethephon treatment

It is well known that plant hormones play a crucial role in plant height regulation ${ }^{[13,71-74]}$. Meristem cell elongation and expansion contribute significantly to the growth and morphogenesis of maize internodes, which usually undergo substantial enlargement when they differentiate. The extent of elongation depends on the cell type and is often regulated by environmental conditions and endogenous hormones.

In this study, three genes (the probe set of $Z m .275 .1$. S1_a_at, Zm.19276.1.A1_at and Zm.7548.1.A1_at) were annotated and related to the ethylene pathway. $\bar{Z} m .275 .1$. S1_a_at, a homolog of Arabidopsis ER-associated receptor and perceives ethylene gas, binds to ethylene and is proposed to inhibit receptor function ${ }^{[10,75]}$. Zm.19276.1. A1_at, the putative homolog of several Arabidopsis EREBP transcription factors, was upregulated 4.92-fold and is known to be the immediate target of EIN3/EIL1, which can bind to a primary ethylene response element in the promoters of EREBP genes ${ }^{[75]}$. Zm.19276.1.A1_at, upregulated 4.92-fold, is the putative homolog of several Arabidopsis EREBP transcription factors, and is known to be the immediate target of EIN3/EIL1, which can bind to a primary ethylene response element in the promoters of EREBP genes ${ }^{[76]}$. Zm.7548.1.A1_at (Opr5) putatively, which encodes a jasmonate-mediated gene related to cyclopentanones, was upregulated 5.35-fold and functions as a plant growth regulator in various developmental processes such as root elongation, senescence, anther dehiscence and tuber formation ${ }^{[77]}$, which are also involved in the ethylene signal transduction pathway.
Two genes (Zm.11809.1.A1_at, Zm.463.1.A1_at) were related to ABA synthesis and signal transduction. It has been reported that the function of the ABA signaling pathway is significantly enriched in response to various biotic and abiotic stresses, seed germination and stomata regulation in various crops ${ }^{[78]}$, but there are, however, few reports of the function of $\mathrm{ABA}$ in relation to plant height or internode elongation. Compared with $\mathrm{ABA}$, although also known as the stress hormone, ethylene has been reported to mediate submergence-induced shoot elongation in rice, which is the initial regulator of fast underwater elongation ${ }^{[79,80]}$. During submergence, low oxygen stresses promote ethylene synthesis in submerged tissues, and elevated GA/IAA and lower ABA levels lead to increases in cell division and cell elongation activities of internodes growth in deep water rice ${ }^{[14]}$. These research findings are completely contrary to our study of maize. This is understandable, as the two crops belong to different species and differ in the evolution of primary process in plants and the developmental responses to their associated physical environments. We speculate that the molecular basis of the internode elongation responses to an ethephon treatment would be different between crop species as different as maize and deep water rice.

Cytokinins are plant-specific hormones that regulate cell division and are the most thoroughly studied hormones involved in maintaining meristem activity ${ }^{[81]}$, and increased cytokinin production is associated with the formation of larger vegetative meristems ${ }^{[82]}$. Based on this study, two probe sets (Zm.325.1.A1_at, Zm.326.1.S1_at) that encode a putative response regulator receiver, identified as ARR homolog genes negatively influencing meristem size, were significantly downregulated ${ }^{[83]}$. The results from previous studies of dwarf mutants indicated that GA plays significant roles in the plant height decision, and plants defective in GA biosynthesis show typical GAdeficient phenotypes, such as dwarfism, small, dark-green leaves, inhibited cell growth and defective flowering ${ }^{[84,85]}$. In maize, several genes have been reported that encode the enzymes involved in GA biosynthesis, and mutants for all of these genes show dramatically reduced plant height ${ }^{[85]}$. $D 8$ is a negative GA-responsive regulator in maize ${ }^{[86]}$, and its orthologs in Arabidopsis $(G A I)^{[87]}$ and wheat $(R h t)^{[88]}$ share a conserved DELLA domain that is important for the recognition of GAs. ZmGA3ox2 (D18), an ortholog of OsGA3ox2, encoding a GA3 $\beta$-hydroxylase, has a similar expression pattern to OsGA3ox2, which has been confirmed as controlling the elongation of the vegetative shoot ${ }^{[89]}$. Here we showed that mRNAs corresponding to four probe sets (Zm.13480.1.S1_at and Zm.390.1.S1_at) were significantly changed in the maize internode elongation responses to ethephon treatment. Zm.13480.1.S1_at, which was upregulated 3.75 times, is an ortholog of OsGA20ox2 (SDI) encoding a gibberellin 20 oxidase, a key enzyme in the biosynthesis of GA that catalyzes the three steps GA $53 \rightarrow$ GA $44 \rightarrow$ GA $19 \rightarrow$ GA 20, which 
has been confirmed as controlling shorter leaves and stems $^{[90]}$.Zm.390.1.S1_at was upregulated 3.30 time, and is an ortholog of $d 3$ (cytochrome P450) encoding an early step in the biosynthesis of GA, probably the 13hydroxylation step, which has been confirmed as regulating shorter plants and stems ${ }^{[91,92]}$. Zm.6485.1.A1_at was downregulated and was identified as a 14-3-3 family protein, which is a post-translational regulator involved in the regulation of endogenous amounts of GA biosynthetic pathway ${ }^{[93]}$.Zm.4871.1.A1_at was downregulated and was identified as growth-regulating factor protein containing two conserved regions: the QLQ (Gln, Leu, Gln) and WRC (Trp, Arg, Cys) domains, which show a pleiotropic phenotypes in Arabidopsis and have regulatory roles in stem elongation, curly leaves and delayed flowing ${ }^{[94]}$. The results indicated that these probe sets involved in GA biosynthetic pathway are likely to lead to a shorter cell phenotype in response to ethylene release, resulting in the reduced maize internode elongation following ethephon treatment.

IAA has also been reported to regulate plant height and cell growth ${ }^{[95]}$. The exogenous application of IAA activates cell division in pericycle cells, resulting in the formation of additional lateral roots ${ }^{[96]}$. In the present data, six probe sets were detected and related to the Aux/IAA signaling pathway. For example, Zm.10147.1.A1_at (NAC transcription factor 49) was significantly upregulated 6.8-fold, and is a putative homolog of an Arabidopsis transcription activator $N A C l$, consisting of an $\mathrm{N}$-terminal conserved NAC-domain that binds to DNA and a Cterminal activation domain, which may form a helix-turnhelix structure that specifically binds target DNA. Previous studies have shown that gene $N A C l$ was induced by auxin and mediates auxin signaling to promote lateral root development ${ }^{[97]}$. Moreover, NAC genes may be critical in adjusting the development of the shoot apical meristem and the differentiation of flower organs, which may participate in incorporating responses to environmental and endogenous stimuli into the process of plant lateral root development ${ }^{[98]}$. The maize ZmSIMK1 gene (Zm.217.1.S1_at) which was significantly upregulated 2.75-fold, encodes mitogen-activated protein kinase 1, which can phosphorylate specific effector proteins leading to activation of cellular responses. ZmSIMK1 has a positive effect in Arabidopsis germination and growth ${ }^{[99]}$. Zm.5439.1.S1_at was significantly downregulated 0.43 -fold, and encodes serine/threonine-protein kinase 12, which is the putative homolog of SNF1-related protein kinase. This kinase was first found in yeast (from where the name originated) and is highly conserved, with pivotal roles in plant growth and metabolic responses to cellular stress $^{[100]}$.Zm.12687.1.S1_a_at (ZmPIN1d), which was significantly downregulated 0.4 -fold, encodes a PIN-form protein. ZmPIN1d was reported to be a PIN auxin efflux carrier family member that is localized in the postembryonic root and expressed in maize root apexes, epidermis, stem and central cylinder ${ }^{[101,102]}$, and marks the transition from the vegetative to the reproductive development in the shoot apical meristem and inflorescence meristem. Therefore, we propose that polar auxin transport decreased in response to released ethylene in maize may trigger a wide range of developmental processes from crosstalk with ethephon treatment, such as inhibiting cell elongation at internodes meristem. c2 (the probe set of Zm.722.1.A1_at) represents a putative homolog of chalcone synthase. Zm.283.1.S1_at was upregulated 0.08 -fold, and encodes maize tryptophan synthase bx1, another metabolic pathway related gene. In our previous study, the endogenous auxin (IAA) and gibberellin (GA4) contents of maize internodes were significantly decreased, abscisic acid (ABA) content was significantly increased, but the ratio of GA4/ABA was significantly decrease ${ }^{[103,104]}$. In combination, this suggests that ethylene release, inducing crosstalk between plant hormone signaling pathways, is one of the explanations for internode shortening and thickening of maize. Moreover, the morphological and microarray data presented here support the suggestion that the potential components of six auxin transport genes and four GA biosynthesis pathway genes play an important role in primarily targeting elongation processes, including upregulation of GA20ox2 and D3, downregulation of General regulatory factor 2 and Growth-regulating factor 1 in the GA signaling pathway, $c 2$, upregulation of MAP kinase 4 and $N A C 1, b x 1$, and downregulation of Auxin efflux carrier component $1 c$, serine/threonine-protein kinase 12 in the Aux/IAA signaling pathway.

Based on a described network of plant hormone signaling pathways ${ }^{[13,68,73,74,105,106]}$ and the putative components of plant hormone responses identified in this research, a putative schematic of the cellular plant hormone signaling pathways in response to ethephon treatment was proposed. The blue probe sets represents the putative cell and cell wall component genes identified in this research. The small arrows indicate up or downregulation of the mRNAs corresponding to the probe sets in response to ethephon treatment. Arrows and t-bars represent positive and negative effects, respectively. Solid lines indicate effects that occur through direct interaction, whereas dotted lines indicate effects that occur through indirect interaction.

\section{Conclusions}

The microarray data showed that more genes are involved in internode elongation $24 \mathrm{~h}$ after ethephon treatment, and many genes in plant hormone signaling pathways were markedly upregulated or downregulated. We also conducted a comparative analysis by qPCR, suggesting that these genes may be important in maize internode responses to gaseous ethylene release. Based on a described network 
of plant hormone signaling pathways ${ }^{[13,73,74,105,106]}$, a putative schematic of the cellular response to ethephon treatment is proposed which incorporates most of the significantly change probe sets identified in maize internodes (Fig. 3). Although little is known about how ethylene triggers such a wide array of diverse responses during the internode elongation process in maize, our results provide a basis for the selection and functional analysis of candidate genes involved in inhibition of internode elongation by ethephon treatment. These include six auxin transport genes and four GA biosynthesis pathway genes, and a gene (the probe set of Zm.13480.1. S1_at), an ortholog gene of OsGA20ox2 (SD1) with an unknown function during internode elongation in response to induced ethylene release in maize. Further functional analyses of the differentially-expressed genes identified in our results should provide more information and eventually a better understanding of the reduction in plant height and prevention of lodging in maize by ethylene.
Acknowledgements This research was supported by the National High Technology Research and Development Program of China (2011AA10 A206). The authors thank Prof. Huub J. Speirtz, Wageningen University, for language improvement.

Supplementary materials The online version of this article at http://dx. doi.org/10.15302/J-FASE-2016103 contains supplementary materials (Appendix A)

Compliance with ethics guidelines Xiaoyi Wei, Weiqiang Zhang, Qian Zhang, Pei Sun, Zhaohu Li, Mingcai Zhang, Jianmin Li, and Liusheng Duan declare that they have no conflict of interest or financial conflicts to disclose.

This article does not contain any studies with human or animal subjects performed by any of the authors.

\section{References}

1. Kang M S, Din A K, Zhang Y, Magari R. Combining ability for rind puncture resistance in maize. Crop Science, 1999, 39(2): 368371

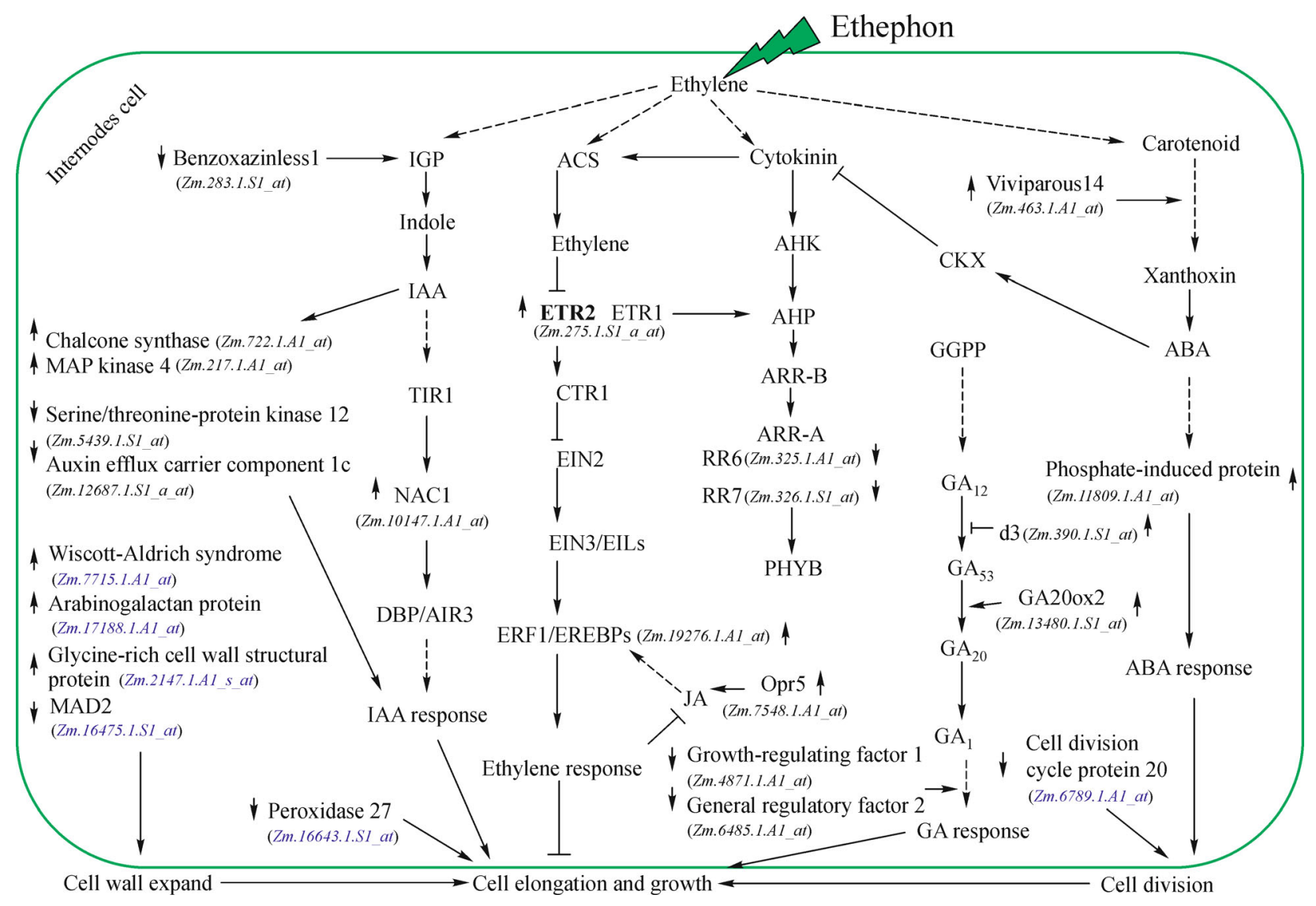

Fig. 3 A putative schematic of the differentially-expressed internode cell genes involved in plant hormone crosstalk in response to ethephon treatment in maize plants. MAD 2, mitotic arrest deficient 2; IGP, indole-3-glycerol phosphate; IAA, indole-3-acetic acid; TIR1, transport inhibitor response 1; NAC1, NaCl stress protein1; DBP, DNA binding protein; AIR3, auxin-induced in root cultures 3; ACS, 1-aminocyclopropane-1-carboxylate synthase; ETR1 (2), ethylene receptor 1 (2); CTR1, constitutive triple response 1; EIN2 (3), ethylene insensitive 2 (3); EILs, ethylene insensitive 3-like; ERF1, ethylene response factor 1; EREBPs: ethylene-responsive element binding protein; AHK, Arabidopsis histidine kinases; AHP, Arabidopsis histidine-phosphotransfer proteins; ARR-A (B), Arabidopsis response regulator-A (B); RR6 (7), response regulator 6 (7); PHYB, phytochromes B; Opr5, 12-oxophytodienoic acid reductase 5; CKX, cytokinin oxidase; GGPP, geranylgeranyl pyrophosphate; GA, gibberellin; ABA, abscisic acid; d3, dwarf plant 3; GA20ox2, gibberellin 20 oxidase 2 . 
2. Mi C, Zhang X, Li S, Yang J, Zhu D, Yang Y. Assessment of environment lodging stress for maize using fuzzy synthetic evaluation. Mathematical and Computer Modelling, 2011, 54(34): $1053-1060$

3. Xue J T, Zhang B M, Dong Z Q, Zhao M, Huang C L. Effects of chemical regulation on lodging and yield of maize. Journal of Maize Sciences, 2009, 2: 024

4. Rajala A, Peltonen-Sainio P. Plant growth regulator effects on spring cereal root and shoot growth. Agronomy Journal, 2001, 93 (4): 936-943

5. Rajala A, Peltonen-Sainio P, Onnela M, Jackson M. Effects of applying stem-shortening plant growth regulators to leaves on root elongation by seedlings of wheat, oat and barley: mediation by ethylene. Plant Growth Regulation, 2002, 38(1): 51-59

6. Shekoofa A, Emam Y. Plant growth regulator (ethepon) alters maize (Zea mays L.) growth, water use and grain yield under water stress. Journal of Agronomy, 2008, 7(1): 41-48

7. Khosravi G R, Anderson I. Growth, yield, and yield components of ethephon-treated corn. Plant Growth Regulation, 1991, 10(1): 2736

8. Merchante C, Alonso J M, Stepanova A N. Ethylene signaling: simple ligand, complex regulation. Current Opinion in Plant Biology, 2013, 16(5): 554-560

9. Habben J E, Bao X, Bate N J, DeBruin J L, Dolan D, Hasegawa D, Helentjaris T G, Lafitte R H, Lovan N, Mo H, Reimann K, Schussler J R. Transgenic alteration of ethylene biosynthesis increases grain yield in maize under field drought-stress conditions. Plant Biotechnology Journal, 2014, 12(6): 685-693

10. Chen J F, Gallie D R. Analysis of the functional conservation of ethylene receptors between maize and Arabidopsis. Plant Molecular Biology, 2010, 74(4-5): 405-421

11. Yamauchi T, Watanabe K, Fukazawa A, Mori H, Abe F, Kawaguchi K, Oyanagi A, Nakazono M. Ethylene and reactive oxygen species are involved in root aerenchyma formation and adaptation of wheat seedlings to oxygen-deficient conditions. Journal of Experimental Botany, 2014, 65(1): 261-273

12. Kendrick M D, Chang C. Ethylene signaling: new levels of complexity and regulation. Current Opinion in Plant Biology, 2008, 11(5): 479-485

13. Stepanova A N, Alonso J M. Ethylene signaling and response: where different regulatory modules meet. Current Opinion in Plant Biology, 2009, 12(5): 548-555

14. Ma B, Chen S, Zhang J. Ethylene signaling in rice. Chinese Science Bulletin, 2010, 55(21): 2204-2210

15. Wang F, Cui X, Sun Y, Dong C H. Ethylene signaling and regulation in plant growth and stress responses. Plant Cell Reports, 2013, 32(7): 1099-1109

16. Gagne J M, Smalle J, Gingerich D J, Walker J M, Yoo S D, Yanagisawa S, Vierstra R D. Arabidopsis EIN3-binding F-box 1 and 2 form ubiquitin-protein ligases that repress ethylene action and promote growth by directing EIN3 degradation. Proceedings of the National Academy of Sciences of the United States of America, 2004, 101(17): 6803-6808

17. Qiao H, Chang K N, Yazaki J, Ecker J R. Interplay between ethylene, ETP1/ETP2 F-box proteins, and degradation of EIN2 triggers ethylene responses in Arabidopsis. Genes \& Development,
2009, 23(4): 512-521

18. Potuschak T, Vansiri A, Binder B M, Lechner E, Vierstra R D, Genschik P. The exoribonuclease XRN4 is a component of the ethylene response pathway in Arabidopsis. Plant Cell Online, 2006, 18(11): 3047-3057

19. Yoo S D, Cho Y H, Tena G, Xiong Y, Sheen J. Dual control of nuclear EIN3 by bifurcate MAPK cascades in $\mathrm{C} 2 \mathrm{H} 4$ signalling. Nature, 2008, 451(7180): 789-795

20. Hahn A, Harter K. Mitogen-activated protein kinase cascades and ethylene: signaling, biosynthesis, or both? Plant Physiology, 2009, 149(3): 1207-1210

21. Kieber J J, Rothenberg M, Roman G, Feldmann K A, Ecker J R. CTR1, a negative regulator of the ethylene response pathway in Arabidopsis, encodes a member of the Raf family of protein kinases. Cell, 1993, 72(3): 427-441

22. Sharp R E, LeNoble M E, Else M A, Thorne E T, Gherardi F. Endogenous ABA maintains shoot growth in tomato independently of effects on plant water balance: evidence for an interaction with ethylene. Journal of Experimental Botany, 2000, 51(350): $1575-1584$

23. Rajala A, Peltonen-Sainio P. Timing applications of growth regulators to alter spring cereal development at high latitudes. Agricultural and Food Science, 2008, 11(3): 233-244

24. Azuma T, Hatanaka T, Uchida N, Yasuda T. Interactions between abscisic acid, ethylene and gibberellin in internodal elongation in floating rice: the promotive effect of abscisic acid at low humidity. Plant Growth Regulation, 2003, 41(2): 105-109

25. LeNoble M E, Spollen W G, Sharp R E. Maintenance of shoot growth by endogenous ABA: genetic assessment of the involvement of ethylene suppression. Journal of Experimental Botany, 2004, 55(395): 237-245

26. Rzewuski G, Sauter M. Ethylene biosynthesis and signaling in rice. Plant Science, 2008, 175(1-2): 32-42

27. Ma T L, Wu W H, Wang Y. Transcriptome analysis of rice root responses to potassium deficiency. BMC Plant Biology, 2012, 12 (1): 161

28. Schnable P S, Hochholdinger F, Nakazono M. Global expression profiling applied to plant development. Current Opinion in Plant Biology, 2004, 7(1): 50-56

29. Zheng J, Fu J J, Gou M Y, Huai J L, Liu Y J, Jian M, Huang Q S, Guo X Y, Dong Z G, Wang H Z, Wang G Y. Genome-wide transcriptome analysis of two maize inbred lines under drought stress. Plant Molecular Biology, 2010, 72(4-5): 407-421

30. Schachtman D P, Shin R. Nutrient sensing and signaling: NPKS. Annual Review of Plant Biology, 2007, 58(1): 47-69

31. Van Zhong G, Burns J K. Profiling ethylene-regulated gene expression in Arabidopsis thaliana by microarray analysis. Plant Molecular Biology, 2003, 53(1): 117-131

32. Cheng W H, Chiang M H, Hwang S G, Lin P C. Antagonism between abscisic acid and ethylene in Arabidopsis acts in parallel with the reciprocal regulation of their metabolism and signaling pathways. Plant Molecular Biology, 2009, 71(1-2): 61-80

33. Hiraga S, Sasaki K, Hibi T, Yoshida H, Uchida E, Kosugi S, Kato T, Mie T, Ito H, Katou S, Seo S, Matsui H, Ohashi Y, Mitsuhara I. Involvement of two rice ETHYLENE INSENSITIVE ${ }_{3}$-LIKE genes in wound signaling. Molecular Genetics and Genomics, 2009, 282 
(5): 517-529

34. Zhu Y, Fu J J, Zhang J P, Liu T S, Jia Z W, Wang J S, Jin Y, Lian Y, Wang M, Zheng J, Hou W, Wang G Y. Genome-wide analysis of gene expression profiles during ear development of maize. Plant Molecular Biology, 2009, 70(1-2): 63-77

35. Xing H, Pudake R N, Guo G, Xing G, Hu Z, Zhang Y, Sun Q, Ni Z. Genome-wide identification and expression profiling of auxin response factor (ARF) gene family in maize. BMC Genomics, 2011, 12(1): 178

36. Sauter M, Kende H. Gibberellin-induced growth and regulation of the cell division cycle in deepwater rice. Planta, 1992, 188(3): 362-368

37. van der Knaap E, Kim J H, Kende H. A novel gibberellin-induced gene from rice and its potential regulatory role in stem growth. Plant Physiology, 2000, 122(3): 695-704

38. Li C, Wong W H. Model-based analysis of oligonucleotide arrays: expression index computation and outlier detection. Proceedings of the National Academy of Sciences of the United States of America, 2001, 98(1): 31-36

39. Livak K J, Schmittgen T D. Analysis of relative gene expression data using real-time quantitative PCR and the $2^{-\Delta \Delta C_{\mathrm{T}}}$ method. Methods, 2001, 25(4): 402-408

40. Kutschera U, Wang Z Y. Growth-limiting proteins in maize coleoptiles and the auxin-brassinosteroid hypothesis of mesocotyl elongation. Protoplasma, 2015, 253(1): 1-12

41. Zhang Q, Cheetamun R, Dhugga K S, Rafalski J A, Tingey S V, Shirley N J, Taylor J, Hayes K, Beatty M, Bacic A, Burton R A, Fincher G B. Spatial gradients in cell wall composition and transcriptional profiles along elongating maize internodes. $B M C$ Plant Biology, 2014, 14(1): 27

42. Bosch M, Mayer C D, Cookson A, Donnison I S. Identification of genes involved in cell wall biogenesis in grasses by differential gene expression profiling of elongating and non-elongating maize internodes. Journal of Experimental Botany, 2011, 62(10): 35453561

43. Goddemeier M L, Wulff D, Feix G. Root-specific expression of a Zea mays gene encoding a novel glycine-rich protein, zmGRP3. Plant Molecular Biology, 1998, 36(5): 799-802

44. Matsuyama T, Satoh H, Yamada Y, Hashimoto T. A maize glycine-rich protein is synthesized in the lateral root cap and accumulates in the mucilage. Plant Physiology, 1999, 120(3): 665674

45. Lv H, Zheng J, Wang T, Fu J, Huai J, Min H, Zhang X, Tian B, Shi Y, Wang G. The maize d2003, a novel allele of VP8, is required for maize internode elongation. Plant Molecular Biology, 2014, 84(3): 243-257

46. Zhang Z, Zhang J, Chen Y, Li R, Wang H, Wei J. Genome-wide analysis and identification of HAK potassium transporter gene family in maize (Zea mays L.). Molecular Biology Reports, 2012, 39(8): 8465-8473

47. Swain S M, Singh D P. Tall tales from sly dwarves: novel functions of gibberellins in plant development. Trends in Plant Science, 2005, 10(3): 123-129

48. Wang Y, Deng D. Molecular basis and evolutionary pattern of GAGID1-DELLA regulatory module. Molecular Genetics and Genomics, 2014, 289(1): 1-9
49. Czechowski T, Bari R P, Stitt M, Scheible W R, Udvardi M K. Real-time RT-PCR profiling of over 1400 Arabidopsis transcription factors: unprecedented sensitivity reveals novel root-and shoot-specific genes. Plant Journal, 2004, 38(2): 366-379

50. Ma J, Morrow D J, Fernandes J, Walbot V. Comparative profiling of the sense and antisense transcriptome of maize lines. Genome Biology, 2006, 7(3): R22

51. Liu X, Fu J, Gu D, Liu W, Liu T, Peng Y, Wang J, Wang G. Genome-wide analysis of gene expression profiles during the kernel development of maize (Zea mays L.). Genomics, 2008, 91 (4): $378-387$

52. Wilson J, Mertens D. Cell wall accessibility and cell structure limitations to microbial digestion of forage. Crop Science, 1995, 35 (1): 251-259

53. Gou J Y, Wang L J, Chen S P, Hu W L, Chen X Y. Gene expression and metabolite profiles of cotton fiber during cell elongation and secondary cell wall synthesis. Cell Research, 2007, 17(5): 422-434

54. Ydenberg C A, Padrick S B, Sweeney M O, Gandhi M, Sokolova O, Goode B L. GMF severs actin-Arp2/3 complex branch junctions by a cofilin-like mechanism. Current Biology, 2013, 23 (12): 1037-1045

55. Levitin B, Richter D, Markovich I, Zik M. Arabinogalactan proteins 6 and 11 are required for stamen and pollen function in Arabidopsis. Plant Journal, 2008, 56(3): 351-363

56. Anand S, Tyagi A K. Characterization of a pollen-preferential gene OSIAGP from rice (Oryza sativa L. subspecies indica) coding for an arabinogalactan protein homologue, and analysis of its promoter activity during pollen development and pollen tube growth. Transgenic Research, 2010, 19(3): 385-397

57. Mangeon A, Junqueira R M, Sachetto-Martins G. Functional diversity of the plant glycine-rich proteins superfamily. Plant Signaling \& Behavior, 2010, 5(2): 99-104

58. Gomez J, Sánchez-Martínez D, Stiefel V, Rigau J, Puigdomènech $\mathrm{P}$, Pagès $\mathrm{M}$. A gene induced by the plant hormone abscisic acid in response to water stress encodes a glycine-rich protein. Nature, 1988, 334(6179): 262-264

59. Elledge S J. Mitotic arrest: Mad2 prevents sleepy from waking up the APC. Science, 1998, 279(5353): 999-1000

60. Yu H G, Muszynski M G, Kelly Dawe R. The maize homologue of the cell cycle checkpoint protein MAD2 reveals kinetochore substructure and contrasting mitotic and meiotic localization patterns. Journal of Cell Biology, 1999, 145(3): 425-435

61. Samac D A, Hironaka C M, Yallaly P E, Shah D M. Isolation and characterization of the genes encoding basic and acidic chitinase in Arabidopsis thaliana. Plant Physiology, 1990, 93(3): 907-914

62. Collinge D B, Kragh K M, Mikkelsen J D, Nielsen K K, Rasmussen U, Vad K. Plant chitinases. Plant Journal, 1993, 3(1): $31-40$

63. Frey M, Stettner C, Paré P W, Schmelz E A, Tumlinson J H, Gierl A. An herbivore elicitor activates the gene for indole emission in maize. Proceedings of the National Academy of Sciences of the United States of America, 2000, 97(26): 14801-14806

64. Xia Z, Turner G C, Hwang C S, Byrd C, Varshavsky A. Amino acids induce peptide uptake via accelerated degradation of CUP9, the transcriptional repressor of the PTR2 peptide transporter. 
Journal ofbiological Chemistry, 2008, 283(43): 28958-28968

65. Dietrich D, Hammes U, Thor K, Suter - Grotemeyer M, Flückiger R, Slusarenko A J, Ward J M, Rentsch D. AtPTR1, a plasma membrane peptide transporter expressed during seed germination and in vascular tissue of Arabidopsis. Plant Journal, 2004, 40(4): 488-499

66. Karim S, Holmström K O, Mandal A, Dahl P, Hohmann S, Brader G, Palva E T, Pirhonen M. AtPTR3, a wound-induced peptide transporter needed for defence against virulent bacterial pathogens in Arabidopsis. Planta, 2007, 225(6): 1431-1445

67. Heller W, Hahlbrock K. Highly purified "flavanone synthase" from parsley catalyzes the formation of naringenin chalcone. Archives of Biochemistry and Biophysics, 1980, 200(2): 617-619

68. Peer W A, Bandyopadhyay A, Blakeslee J J, Makam S N, Chen R $\mathrm{J}$, Masson P H, Murphy A S. Variation in expression and protein localization of the PIN family of auxin efflux facilitator proteins in flavonoid mutants with altered auxin transport in Arabidopsis thaliana. Plant Cell Online, 2004, 16(7): 1898-1911

69. Franken P, Niesbach-Klösgen U, Weydemann U, MarechalDrouard L, Saedler H, Wienand U. The duplicated chalcone synthase genes $\mathrm{C} 2$ and Whp (white pollen) of Zea mays are independently regulated; evidence for translational control of Whp expression by the anthocyanin intensifying gene in. $E M B O$ Journal, 1991, 10(9): 2605

70. Taylor L P, Briggs W R. Genetic regulation and photocontrol of anthocyanin accumulation in maize seedlings. Plant Cell Online, 1990, 2(2): 115-127

71. Della Vedova C B, Lorbiecke R, Kirsch H, Schulte M B, Scheets K, Borchert L M, Scheffler B E, Wienand U, Cone K C, Birchler J A. The dominant inhibitory chalcone synthase allele C2-Idf (Inhibitor diffuse) from Zea mays (L.) acts via an endogenous RNA silencing mechanism. Genetics, 2005, 170(4): 1989-2002

72. Wang Y, Li J. Molecular basis of plant architecture. Annual Review of Plant Biology, 2008, 59(1): 253-279

73. Depuydt S, Hardtke C S. Hormone signalling crosstalk in plant growth regulation. Current Biology, 2011, 21(9): R365-R373

74. Peer W A. From perception to attenuation: auxin signalling and responses. Current Opinion in Plant Biology, 2013, 16(5): 561568

75. Gallie D, Young T. The ethylene biosynthetic and perception machinery is differentially expressed during endosperm and embryo development in maize. Molecular Genetics and Genomics, 2004, 271(3): 267-281

76. Guo H, Ecker J R. The ethylene signaling pathway: new insights. Current Opinion in Plant Biology, 2004, 7(1): 40-49

77. Zhang J, Simmons C, Yalpani N, Crane V, Wilkinson H, Kolomiets M. Genomic analysis of the 12-oxo-phytodienoic acid reductase gene family of Zea mays. Plant Molecular Biology, 2005, 59(2): 323-343

78. Speirs J, Binney A, Collins M, Edwards E, Loveys B. Expression of ABA synthesis and metabolism genes under different irrigation strategies and atmospheric VPDs is associated with stomatal conductance in grapevine (Vitis vinifera L. cv Cabernet Sauvignon). Journal of Experimental Botany, 2013, 64(7): 1907-1916

79. Fukao T, Bailey-Serres J. Submergence tolerance conferred by Sub1A is mediated by SLR1 and SLRL1 restriction of gibberellin responses in rice. Proceedings of the National Academy of Sciences of the United States of America, 2008, 105(43): 16814-16819

80. Jackson M B. Ethylene-promoted elongation: an adaptation to submergence stress. Annals of Botany, 2008, 101(2): 229-248

81. Riou-Khamlichi C, Huntley R, Jacqmard A, Murray J A. Cytokinin activation of Arabidopsis cell division through a D-type cyclin. Science, 1999, 283(5407): 1541-1544

82. Rupp H M, Frank M, Werner T, Strnad M, Schmülling T. Increased steady state mRNA levels of the STM and KNAT1 homeobox genes in cytokinin overproducing Arabidopsis thaliana indicate a role for cytokinins in the shoot apical meristem. Plant Journal, 1999, 18(5): 557-563

83. Leibfried A, To J P, Busch W, Stehling S, Kehle A, Demar M, Kieber J J, Lohmann J U. WUSCHEL controls meristem function by direct regulation of cytokinin-inducible response regulators. Nature, 2005, 438(7071): 1172-1175

84. Fleet C M, Sun T P. A DELLAcate balance: the role of gibberellin in plant morphogenesis. Current Opinion in Plant Biology, 2005, 8 (1): $77-85$

85. Wang Y, Deng D, Ding H, Xu X, Zhang R, Wang S, Bian Y, Yin Z, Chen Y. Gibberellin biosynthetic deficiency is responsible for maize dominant Dwarf11 (D11) mutant phenotype: physiological and transcriptomic evidence. PLoS ONE, 2013, 8(6): e66466

86. Winkler R G, Freeling M. Physiological genetics of the dominant gibberellin-nonresponsive maize dwarfs, Dwarf8 and Dwarf9. Planta, 1994, 193(3): 341-348

87. Peng J, Carol P, Richards D E, King K E, Cowling R J, Murphy G P, Harberd N P. The Arabidopsis GAI gene defines a signaling pathway that negatively regulates gibberellin responses. Genes \& Development, 1997, 11(23): 3194-3205

88. Gale M D, Devos K M. Comparative genetics in the grasses. Proceedings of the National Academy of Sciences of the United States of America, 1998, 95(5): 1971-1974

89. Teng F, Zhai L, Liu R, Bai W, Wang L, Huo D, Tao Y, Zheng Y, Zhang Z. ZmGA3ox2, a candidate gene for a major QTL, $q P H 3.1$, for plant height in maize. Plant Journal, 2013, 73(3): 405-416

90. Spielmeyer W, Ellis M H, Chandler P M. Semidwarf (sd-1), “green revolution" rice, contains a defective gibberellin 20-oxidase gene. Proceedings of the National Academy of Sciences of the United States of America, 2002, 99(13): 9043-9048

91. Winkler R G, Helentjaris T. The maize Dwarf3 gene encodes a cytochrome P450-mediated early step in Gibberellin biosynthesis. Plant Cell Online, 1995, 7(8): 1307-1317

92. Jiang F, Guo M, Yang F, Duncan K, Jackson D, Rafalski A, Wang $\mathrm{S}$, Li B. Mutations in an AP2 transcription factor-like gene affect internode length and leaf shape in maize. PLoS ONE, 2012, 7(5): e37040

93. Igarashi D, Ishida S, Fukazawa J, Takahashi Y. 14-3-3 proteins regulate intracellular localization of the bZIP transcriptional activator RSG. Plant Cell Online, 2001, 13(11): 2483-2497

94. Kim J H, Choi D, Kende H. The AtGRF family of putative transcription factors is involved in leaf and cotyledon growth in Arabidopsis. Plant Journal, 2003, 36(1): 94-104

95. Mockaitis K, Estelle M. Auxin receptors and plant development: a new signaling paradigm. Annual Review of Cell and Developmental Biology, 2008, 24(1): 55-80 
96. Laskowski M J, Williams M E, Nusbaum H C, Sussex I M. Formation of lateral root meristems is a two-stage process. Development, 1995, 121(10): 3303-3310

97. Xie Q, Frugis G, Colgan D, Chua N H. Arabidopsis NAC1 transduces auxin signal downstream of TIR1 to promote lateral root development. Genes \& Development, 2000, 14(23): 3024 3036

98. He X J, Mu R L, Cao W H, Zhang Z G, Zhang J S, Chen S Y. AtNAC2, a transcription factor downstream of ethylene and auxin signaling pathways, is involved in salt stress response and lateral root development. Plant Journal, 2005, 44(6): 903-916

99. Gu L, Liu Y, Zong X, Liu L, Li D P, Li D Q. Overexpression of maize mitogen-activated protein kinase gene, ZmSIMK1 in Arabidopsis increases tolerance to salt stress. Molecular Biology Reports, 2010, 37(8): 4067-4073

100. Hrabak E M, Chan C W, Gribskov M, Harper J F, Choi J H, Halford N, Kudla J, Luan S, Nimmo H G, Sussman M R. The Arabidopsis CDPK-SnRK superfamily of protein kinases. Plant Physiology, 2003, 132(2): 666-680
101. Knöller A S, Blakeslee J J, Richards E L, Peer W A, Murphy A S. Brachytic2/ZmABCB1 functions in IAA export from intercalary meristems. Journal of Experimental Botany, 2010, 61(13): 36893696

102. McLamore E S, Diggs A, Calvo Marzal P, Shi J, Blakeslee J J, Peer W A, Murphy A S, Porterfield D M. Non-invasive quantification of endogenous root auxin transport using an integrated flux microsensor technique. Plant Journal, 2010, 63(6): 1004-1016

103. Wei X Y, Zhang M C, Li Z H, Duan L S. Differences in responding sensitivity to ethephon among different maize genotypes. Acta Agronomica Sinica, 2011, 37(10): 1819-1827

104. Wei X Y, Zhang M C, Zhang Y, Li Z H, Duan L S. Effects of ethephon on internode elongation and endogenous hormones in different genotypes of maize. Chinese Journal of Pesticide Science, 2011, 13(5): 475-479

105. Werner T, Schmulling T. Cytokinin action in plant development. Current Opinion in Plant Biology, 2009, 12(5): 527-538

106. Daviere J M, Achard P. Gibberellin signaling in plants. Development, 2013, 140(6): 1147-1151 